\title{
Environmental radioactivity of pegmatites associating younger granite of Ras Baroud area, Central Eastern Desert, Egypt.
}

\author{
Sameh Z. Tawfik \\ Nuclear Materials Authority, Cairo, Egypt. \\ samehzakaria@outlook.com
}

\begin{abstract}
Ras Baroud pluton is one of the well exposed granitic batholiths in the Central Eastern Desert of Egypt. The granites occure as isolated plutons of high relief circular and semicircular outlines. They are cut by zoned pegmatites and dikes of different types. The pegmatites are found as lenticular pockets and sheet-like bodies along the southern periphery of Ras Baroud pluton. The zonal sequence of the pegmatites is formed of an outer blocky feldspar zone. The intermediate mica zone (muscovite and/or biotite) and an inner zone of massive quartz might present in some pockets and absent in others. In some pegmatite pockets, the intermediate zone of mica occurs with some distinct dark alteration parts that record the highest radioactivity levels. The geochemical studies revealed that these pegmatites are attributed to low temperature late differentiated magmatic fluids enriched in volatiles.

Field radiometric survey was used to evaluate the suitability of mineral resources at the different pegmatite zones to industrial use. The environmental radioactive parameters showed that the radioactivity of all four zones of the pegmatites is higher than the safety limit which indicate hazardous effects on human lives if used as indore building and/or ornamental stones. Also, the uranium migration rates and directions showed that uranium migrates from the radioactive altered zone which has the higher uranium content towards the other three pegmatite zones (outer, intermediate and inner zones).

From a paragenetic point of view, zones in pegmatite are developed from the wall inward within a restricted pegmatitic stage. In the early stage, zoned pegmatites of simple mineralogy are produced. In the later stage, residual fluids rich in soda, silica, alumina and significant quantities of $\mathrm{Nb}, \mathrm{Ta}, \mathrm{U}, \mathrm{Th}$ and $\mathrm{F}$ travel along fractures, react with the previously formed pegmatitic minerals forming new minerals of replacement origin.

The zoned pegmatites host U-minerals (betafite and kasolite) as well as $\mathrm{Nb}$ Ta mineral (columbite) as invisible disseminations in the intermediate alteration zone, that confines their formation in a temperature range between 300 and $500^{\circ} \mathrm{C}$. The hydrothermal activity and the alteration processes are responsible for the deposition of the uranium minerals.
\end{abstract}

Keywords: Environment, Baroud, pegmatite, radioactivity, uranium, columbite, betafite, kasolite.

\section{INTRODUCTION}

Uranium exploration activities in Egypt, led to the discovery of several uranium occurrences and high radiometric anomalies. These uranium occurrences sometimes have economic minerals which might carry radioactive elements in its lattice. In most of 
these occurrences, the U-mineralizations are structurally controlled with preferable development at the marginal zones of their enclosing granites and pegmatites (Assaf et al., 1997) or associated with wide scale alteration features (Shalaby and Moharem, 2001). The alteration and fracturing of U-rich granites aided meteoric water and hydrothermal solutions to liberate labile uranium and later precipitate their loads along micro-fractures, joints and fault planes (Scheepers, 2000).

The present work aims to use of environmental radioactivity for evaluating the safety of zoned pegmatites mineral resources industrial use for both workers and consumers. A well calibrated RS-230 field spectrometer was used for spectrometric inspection of the zoned pegmatite layers (outer feldspar, intermediate mica, alteration and inner quartz zones). These readings were used to calculate environmental parameters that gave us a clear view about the uranium contents and uranium migration directions among the layers of zoned pegmatite in order to understand how much uranium goes through (in and/or out) these zones.

\section{GEOLOGIC OUTLINES}

Gabal Ras Baroud area is located in the Central Eastern Desert of Egypt between latitudes $26^{\circ} 44^{\circ}$ and $26^{\circ} 50^{\circ} \mathrm{N}$ and longitudes $33^{\circ} 35^{\circ}$ and $33^{\circ} 42^{\circ} \mathrm{E}$ (Fig. 1). It has a semicircular mass covering an area of about $40 \mathrm{~km}^{2}$. The pluton forms a prominent topographic feature rising as high as $1446 \mathrm{~m}$ above sea level. The contacts with the surrounding rocks are almost sharp. These rocks are jointed, faulted and traversed by basaltic and post granitic dikes and veins of pegmatites and quartz (Omar, 1995 and Abd El Warith, 2000). These dikes occur as parallel swarms trending in the WNW-ESE direction with steep dips towards the north (Mahdy et al., 1994; Omar, 1995 and Abd El Warith, 2000).

Abdel Monsif et al., 2018 stated that Ras Baroud younger granites are medium- to coarse-grained, pink to reddish pink in colour, equigranular, hypidiomorphic rocks. They are essentially composed of orthoclase perthite, quartz, plagioclase and muscovite, in addition to subsidiary biotite. The accessory and secondary minerals include; zircon, titanite, apatite, fluorite, epidote and iron oxy-hydroxide minerals. 


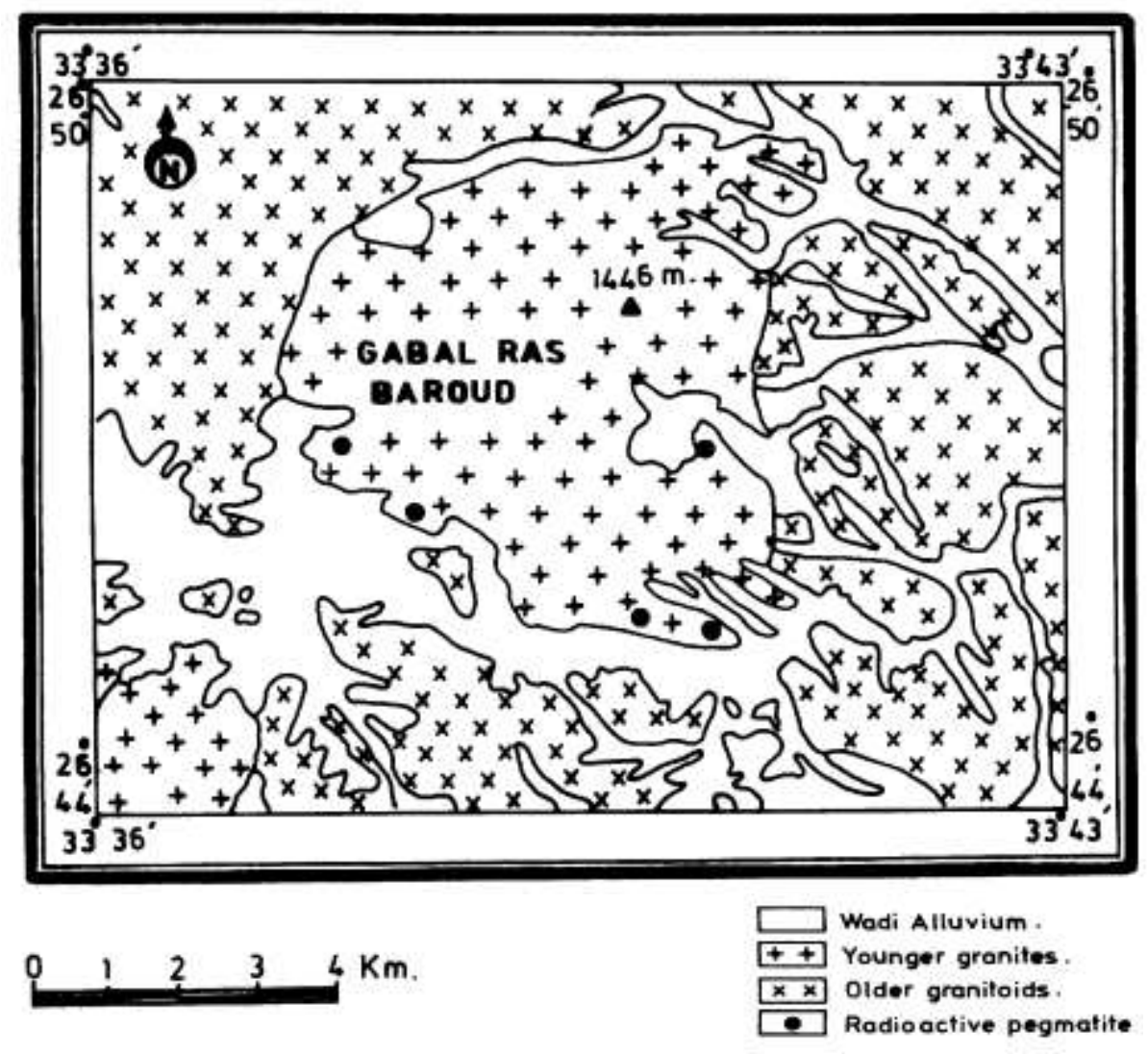

Fig. (1): Geological map of Gabal Ras Baroud, Central Eastern Desert, Egypt. (Modified after Omar, 1995 and Abd El Warith, 2000).

At Gabal Ras Baroud, the granite pluton host pegmatite bodies that occur as lenticular pockets and sheet-like bodies, varying in size between 0.3 and $30 \mathrm{~cm}$ in width and from 0.6 to $50 \mathrm{~cm}$ in length. They occur as zoned and unzoned pegmatite bodies. The zoned pegmatites are located along the southern periphery of the pluton (Fig. 2). They consist of an outer zone of blocky feldspars with subordinate randomly distributed mica pockets (as intermediate zone) and an inner core of massive quartz. Some intermediate zones have altered parts often along fractures and in contact with quartz cores. However, the unzoned type dominates as large lenticular or circular pockets at the higher altitude of the pluton.

The pegmatitic bodies in the granitic mass of Gabal Ras Baroud are associating Nb-Ta anomalies. The previous studies of Mahdy, et al., (1994); Omar, (1995) and Abd El Warith, (2000) suggest that the anomalous values of $\mathrm{Nb}$ and Ta can be attributed to the dissemination of their own minerals. The $\mathrm{Nb}$ - $\mathrm{Ta}$ mineralizations occur as clusters of fan-shaped megascopic long prismatic columbite crystals (up to $11 \mathrm{~cm}$ long) associated with anomalous radioactive invisible disseminations in the outer zone of the feldspar. 


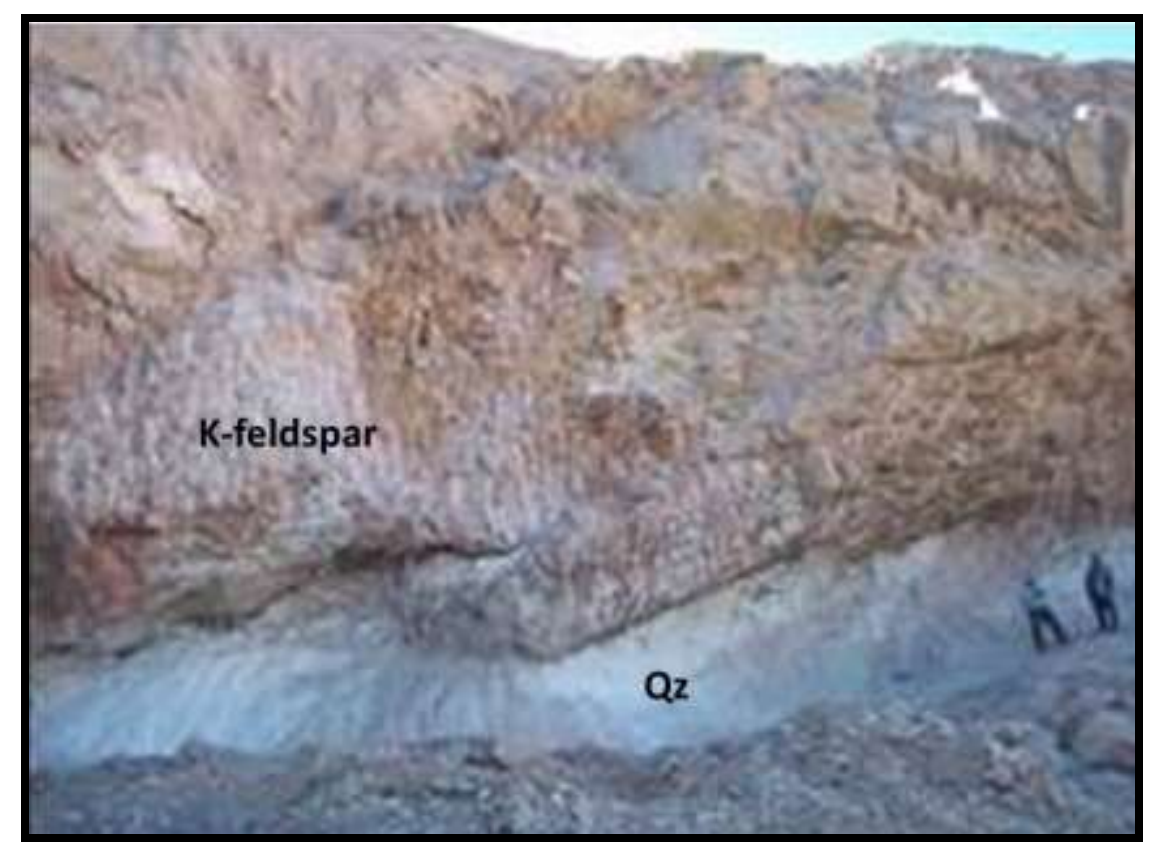

Fig. (2): Photomicrograph showoing the zones of pegmatite at Gabal Ras Baroud granitic pluton.

However, there are two types of pegmatitic rocks in the area of study namely; zoned and unzoned. The unzoned pegmatites are mainly composed of microperthite ( $20 \%$ orthoclase perthite and $80 \%$ microcline perthite), quartz, plagioclase, muscovite and biotite as essential minerals. Opaques, bastnasite, fluorite, titanite and zircon represent the accessory minerals. The zoned pegmatites are composed of thick part of coarse grained feldspars as outer zone and inner zone of quartz with or without intermediate zone of mica, and less commonly some important accessory mineral.

Generally, the granitic border zone of Ras Baroud pluton is concealed in most cases. However, the exposed parts are represented by gradational contact between the outer zone of the feldspar and country rocks (younger granites). The essential mineral composition of pegmatite includes; quartz, orthoclase, microcline perthites, plagioclase and mica (muscovite and/or biotite). The accessory minerals are fluorite, zircon and hematite.

\section{METHODOLOGY}

Thirty field radioactivity readings were recorded at each zone (outer, intermediate, altered and inner zones) of the zoned pegmatite. The ${ }^{238} \mathrm{U}(\mathrm{ppm}),{ }^{232} \mathrm{Th}$ $(\mathrm{ppm})$ and ${ }^{40} \mathrm{~K}(\%)$ were recorded for each station in order to calculate the environmental radioactivity impact parameters for each zone. One of the main objectives of environmental radioactivity measurements is not only to determine the activity concentrations of ${ }^{238} \mathrm{U},{ }^{232} \mathrm{Th}$ and ${ }^{40} \mathrm{~K}$, but also to estimate the radiation exposure 
dose and to assess the biological effects on human. This involves an estimate of the radiological dose (or radiation energy) that may be absorbed by a potentially exposed individual as a result of specific exposure (Beretka and Matthew, 1985) (UNSCEAR, 1998). The field radiometric survey of Gabal Ras Baroud area was accomplished using the portable gamma-ray spectrometer (RS-230).

\section{III.1. Environmental Radioactivity Parameters}

Rocks used in some industrial applications such as utilization of these rocks as an ornamental stones and in building materials must get tested for their suitability and safety. Physico-mechanical characterization detected to determine their suitability for uses as an ornamental stones. All rocks passes the tests are suitable as ornamental stones (Lasheen, 2019).

\section{Absorbed Dose Rate in Air (D)}

The absorbed gamma dose rates in air at $1 \mathrm{~m}$ above the ground surface for the uniform distribution of radionuclides $\left({ }^{238} \mathrm{U},{ }^{232} \mathrm{Th}\right.$ and $\left.{ }^{40} \mathrm{~K}\right)$ were calculated by using Equation 1 on the basis of guide lines provided by UNSCEAR (2000) and Örgün et al. (2007).

$$
D(n G y / h)=0.462 A_{U}+0.604 A_{T h}+0.0417 A_{K}
$$

where $A_{U}, A_{T h}$ and $A_{K}$ are the average specific activities of ${ }^{226} \mathrm{Ra},{ }^{232} \mathrm{Th}$ and ${ }^{40} \mathrm{~K}$ in $\mathrm{Bq} / \mathrm{kg}$, respectively.

\section{Annual Effective Dose Equivalent (AEDE)}

The annual effective dose equivalent (AEDE) was calculated from the absorbed dose by applying the dose conversion factor of $0.7 \mathrm{~Sv} / \mathrm{Gy}$ and the outdoor occupancy factor of 0.2 (UNSCEAR, 2000; Örgün et al. 2007).

\section{Radium Equivalent Activity (Raeq)}

The radium equivalent activity for the samples was calculated. The exposure to radiation (Tufail et al. 1992) can be defined in terms of the radium equivalent activity $\left(\mathrm{Ra}_{\mathrm{eq}}\right)$, which can be expressed by the following equation:

$$
\operatorname{Ra}_{\mathrm{eq}}=\mathrm{A}_{\mathrm{Ra}}+\mathbf{1 0} / 7 \mathrm{~A}_{\mathrm{Th}}+10 / 130 \mathrm{~A}_{K}
$$

where $A_{R a}, A_{T h}$ and $A_{K}$ are the specific activities of Ra, Th and $K$, respectively, in $B q$ $\mathrm{kg}^{1}$. 


\section{External and Internal Hazard Index $\left(H_{e x}\right.$ and $\left.H_{\text {in }}\right)$}

To limit the annual external gamma-ray dose (Saito and Jacob, 1995 and UNSCEAR, 2000) to 1.5 Gy for the samples under investigation, the external hazard index $\left(\mathrm{H}_{\mathrm{ex}}\right)$ is given by the following equation:

$$
H_{\text {ex }}=A_{U} / 370+A_{T h} / 259+A_{K} / 4810
$$

The internal exposure to ${ }^{222} \mathrm{Rn}$ and its radioactive progeny is controlled by the internal hazard index $\left(\mathrm{H}_{\mathrm{in}}\right)$, which is given given by Nada (2003).

$$
H_{\text {in }}=A_{U} / 185+A_{T h} / 259+A_{K} / 4810
$$

These indices must be less than unity in order to keep the radiation hazard insignificant (Lakehal et al., 2010; Baykara et al., 2010).

\section{Activity Concentration Index (Iy)}

Another radiation hazard index called the representative level index, $\mathbf{I} \boldsymbol{\gamma}$, is defined as follows (NEA-OECD, 1979):

$$
I_{\gamma}=\mathrm{Au}_{\mathrm{U}} / \mathbf{1 5 0}+\mathrm{A}_{\mathrm{Th}} / \mathbf{1 0 0}+\mathrm{AK}_{\mathrm{K}} / \mathbf{1 5 0 0}
$$

where $A_{U}, A_{T h}$ and $A_{K}$ are the activity concentrations of ${ }^{226} \mathrm{Ra},{ }^{232} \mathrm{Th}$ and ${ }^{40} \mathrm{~K}$, respectively in $\mathrm{Bq} \mathrm{kg}^{-1}$ (Abbady et. al., 2005). The safety value for this index is $\leq 1$ (El Galy et al., 2008; El Aassy et al., 2012; Harpy et al. 2019b; Taha et al. 2020). Tables 1, 2, 3 and 4 show the environmental radioactivity parameters for the studied pegmatite zones (outer, intermediate, altered and inner).

The average absorbed $\gamma$ - dose rate (D) values for the studied zones of Ras Baroud pegmatites are shown in tables $3,4,5 \& 6$. The calculated values of the studied zones are 540, 297, 2269 and $224 \mathrm{nGyh}^{-1}$ for outer, intermediate, altered and inner zones respectively. These estimated values of absorbed $\gamma$ - dose rate in the studied samples are comparably higher than the world average value $57 \mathrm{nGyh}^{-1}$ (Harpy et al. 2019b; Taha et al. 2020). This indicates the presence of harmful effects.

The annual effective dose for the studied pegmatite zones varied from 76, 42, 318 and $31 \mathrm{mSvy}^{-1}$ for outer, intermediate, altered and inner zones respectively. All mean values of the four zones of pegmatite are higher than the worldwide average of the annual effective dose $0.48 \mathrm{mSvy}^{-1}$ recommended by UNSCEAR (2000). This indicates that none of the pegmatite zones satisfy the criteria for radiation safety.

The radium equivalent activity $\mathrm{Ra}_{\mathrm{eq}}$ for the studied pegmatite zones ranges from 793, 479 and $3194 \mathrm{~Bq} \mathrm{Kg}^{-1}$ (outer, intermediate and altered and inner zones respectively) 
which was to some extent higher than the maximum permitted value (370 Bq Kg-1). The inner zone only has relatively lower radium equivalent activity and $291 \mathrm{~Bq} \mathrm{Kg}^{-1}$.

The values of external hazard indices $\left(\mathrm{H}_{\text {in }}\right)$ for the four zones of pegmatite ranges between 3.2,1.7,13,6 and 1.3, while the internal hazard index varies between 5.3, 3, 22 and 2 for the outer, intermediate, altered and inner zones respectively. External and internal hazard indices were significantly higher than unity for most of the recorded readings suggesting that these rocks couldn't be used as building and interior decorative material of dwelling.

The gamma activity index (I $\gamma$ ) used to estimate gamma-ray hazardous activity associated with the natural radionuclides in the studied pegmatite zones. The obtained values for the inspected pegmatite zones range between 8, 4, 34 and 3 for the outer, intermediate, altered and inner zones respectively. The obtained values of gamma activity indices in all zones have higher doses criterion $(0.3 \mathrm{mSv} / \mathrm{y})$ and/or higher than an activity concentration index of $2 \leq \mathrm{I} \gamma \leq 6$ for materials used in bulk construction except the intermediate mica and inner quartz zones.

\section{III.2. Uranium mobilization}

Type (migration in or migration out a certain rock unit) and amount of the mobilized uranium as well as the uranium mobilization rate of the studied rocks are determined and calculated through several steps using equations of Benzing Uranium Institute of China (1977) and CNNC (1993) as follows:

-Calculate the paleo-uranium background (the original uranium content):

$\mathrm{Uo}=\mathrm{eTh} * \mathrm{eU} / \mathrm{eTh}$

Where Uo is the original uranium content, eTh is the average of thorium content in certain geologic unit and eU/eTh is the average of the regional eU/eTh ratio in different geologic units.

- Calculate the amount of migrated uranium

$$
\mathrm{Um}=\mathrm{Up}-\mathrm{UO}
$$

Where Um is the amount of the mobilized uranium and Up is the average of the present uranium content in certain geologic unit.

Two states of Um are expected; i) if $U m>0$, this means that $U$ could be gained or mobilized into the geologic body during late evolution (migration in) and ii) if $\mathrm{Um}<0$, this indicates $U$ could be lost from the geologic body during late evolution (migration out). 
Table (1): Environmental radioactivity parameters of the outer feldspar zone.

\begin{tabular}{|c|c|c|c|c|c|c|c|c|c|c|c|c|}
\hline Sample No. & $\begin{array}{c}\mathrm{eU} \\
(\mathrm{ppm})\end{array}$ & $\begin{array}{c}\text { eTh } \\
(\mathbf{p p m})\end{array}$ & \begin{tabular}{|c|}
$\mathbf{K}$ \\
$(\%)$
\end{tabular} & $\begin{array}{c}\mathrm{eU} \\
\mathrm{Bq} / \mathrm{kg}\end{array}$ & $\begin{array}{c}\text { eTh } \\
\text { Bq/kg }\end{array}$ & $\begin{array}{c}\mathrm{K} \\
\mathrm{Bq} / \mathrm{kg}\end{array}$ & $\begin{array}{c}\text { Abs. Dose } \\
{ }^{238} \text { U(nGy/h) }\end{array}$ & $\begin{array}{l}\text { Eff. Dose } \\
(\mathrm{mSv})\end{array}$ & Raeq & $H_{\text {ex }}$ & $H_{\text {in }}$ & $\boldsymbol{I}_{\gamma}$ \\
\hline 1 & 67.9 & 58 & 1.6 & 839 & 235 & 496 & 550.33 & 77.05 & 838.57 & 3.28 & 5.55 & 8.28 \\
\hline 2 & 67.5 & 55 & 2.7 & 834 & 223 & 837 & 554.91 & 77.69 & 833.63 & 3.29 & 5.54 & 8.35 \\
\hline 3 & 66.6 & 52 & 3.8 & 823 & 211 & 1178 & 556.64 & 77.93 & 822.52 & 3.28 & 5.51 & 8.38 \\
\hline 4 & 66.5 & 56 & 2.6 & 821 & 227 & 806 & 550.36 & 77.05 & 821.28 & 3.27 & 5.48 & 8.29 \\
\hline 5 & 66.4 & 53 & 1.9 & 820 & 215 & 589 & 533.39 & 74.67 & 820.05 & 3.17 & 5.39 & 8.01 \\
\hline 6 & 66.2 & 51 & 2.5 & 818 & 207 & 775 & 535.10 & 74.91 & 817.58 & 3.17 & 5.38 & 8.04 \\
\hline 7 & 65.7 & 56 & 3.4 & 811 & 227 & 1054 & 556.14 & 77.86 & 811.40 & 3.29 & 5.48 & 8.39 \\
\hline 8 & 65.6 & 53 & 3 & 810 & 215 & 930 & 543.04 & 76.03 & 810.17 & 3.21 & 5.40 & 8.17 \\
\hline 9 & 65.5 & 56 & 3.1 & 809 & 227 & 961 & 551.12 & 77.16 & 808.93 & 3.26 & 5.45 & 8.31 \\
\hline 10 & 65.5 & 54 & 1.7 & 809 & 219 & 527 & 528.12 & 73.94 & 808.93 & 3.14 & \begin{tabular}{|l|l|}
5.33 \\
\end{tabular} & 7.94 \\
\hline 11 & 65.2 & 56 & 3.3 & 805 & 227 & 1023 & 552.00 & 77.28 & 805.23 & 3.27 & 5.44 & 8.32 \\
\hline 12 & 64.9 & 55 & 4.3 & 802 & 223 & 1333 & 560.76 & 78.51 & 801.52 & 3.31 & 5.47 & 8.47 \\
\hline 13 & 64.5 & 59 & 1.4 & 797 & 240 & 434 & 530.80 & 74.31 & 796.58 & 3.17 & 5.32 & 8.00 \\
\hline 14 & 64.3 & 57 & 2.9 & 794 & 231 & 899 & 544.14 & 76.18 & 794.11 & 3.23 & 5.37 & 8.21 \\
\hline 15 & 63.7 & 59 & 2.6 & 787 & 240 & 806 & 541.75 & 75.84 & 786.70 & 3.22 & 5.34 & 8.18 \\
\hline 16 & 63.6 & 57 & 4.9 & 785 & 231 & 1519 & 566.00 & 79.24 & 785.47 & 3.33 & 5.46 & 8.56 \\
\hline 17 & 63.6 & 57 & 1.6 & 785 & 231 & 496 & 523.34 & 73.27 & 785.47 & 3.12 & 5.24 & 7.88 \\
\hline 18 & 63.6 & 55 & 3.9 & 785 & 223 & 1209 & 548.17 & 76.74 & 785.47 & 3.24 & 5.36 & 8.28 \\
\hline 19 & 63.5 & 55 & 3.3 & 784 & 223 & 1023 & 539.84 & 75.58 & 784.23 & 3.19 & 5.31 & 8.14 \\
\hline 20 & 63.1 & 54 & 2.9 & 779 & 219 & 899 & 529.94 & 74.19 & \begin{tabular}{|l|}
779.29 \\
\end{tabular} & 3.14 & 5.25 & 7.99 \\
\hline 21 & 63.1 & 51 & 1.5 & 779 & 207 & 465 & 504.48 & 70.63 & 779.29 & 3.00 & 5.11 & 7.58 \\
\hline 22 & 62.8 & 52 & 2.5 & 776 & 211 & 775 & 518.15 & 72.54 & \begin{tabular}{|l|}
775.59 \\
\end{tabular} & 3.07 & 5.17 & 7.80 \\
\hline 23 & 62.5 & 53 & 2.8 & 772 & 215 & 868 & 522.77 & 73.19 & 771.88 & 3.10 & 5.18 & 7.88 \\
\hline 24 & 62.5 & 52 & 2.9 & 772 & 211 & 899 & 521.61 & 73.03 & 771.88 & 3.09 & 5.17 & 7.86 \\
\hline 25 & 62.4 & 56 & 3 & 771 & 227 & 930 & 532.14 & 74.50 & 770.65 & 3.15 & 5.24 & 8.03 \\
\hline 26 & 62.3 & 59 & 4.2 & 769 & 240 & 1302 & 554.44 & 77.62 & 769.41 & 3.28 & 5.35 & 8.39 \\
\hline 27 & 62.2 & 55 & 3.4 & 768 & 223 & 1054 & 533.72 & 74.72 & 768.18 & 3.16 & 5.23 & 8.06 \\
\hline 28 & 62 & 58 & 3.8 & 766 & 235 & 1178 & 545.11 & 76.31 & 765.71 & 3.22 & 5.29 & 8.24 \\
\hline 29 & 62 & 58 & 2.3 & 766 & 235 & 713 & 525.72 & 73.60 & \begin{tabular}{|l|}
765.71 \\
\end{tabular} & 3.13 & 5.20 & 7.93 \\
\hline 30 & 62 & 56 & 3.8 & 766 & 227 & 1178 & 540.20 & 75.63 & 765.71 & 3.19 & 5.26 & 8.16 \\
\hline Average & 64.24 & 55.27 & 2.92 & 793.36 & 224.38 & 905.20 & 539.81 & 75.57 & 793.37 & 3.20 & 5.34 & 8.14 \\
\hline
\end{tabular}


Table (2): Environmental radioactivity parameters of the intermediate mica zone.

\begin{tabular}{|c|c|c|c|c|c|c|c|c|c|c|c|c|}
\hline $\begin{array}{l}\text { Sample } \\
\text { No. }\end{array}$ & $\left.\begin{array}{c}\mathrm{eU} \\
(\mathbf{p p m})\end{array}\right)$ & \begin{tabular}{|c|} 
eTh \\
$(\mathbf{p p m})$
\end{tabular} & $\begin{array}{c}\mathrm{K} \\
(\%)\end{array}$ & \begin{tabular}{|c|}
$\mathrm{eU}$ \\
$\mathrm{Bq} / \mathrm{kg}$
\end{tabular} & \begin{tabular}{|l} 
eTh \\
Bq/kg
\end{tabular} & $\begin{array}{c}\mathrm{K} \\
\mathrm{Bq} / \mathrm{kg}\end{array}$ & $\begin{array}{c}\text { Abs. Dose } \\
{ }^{238} \mathrm{U}(\mathrm{nGy} / \mathrm{h})\end{array}$ & $\begin{array}{c}\text { Eff. Dose } \\
(\mathrm{mSv})\end{array}$ & $\mathbf{R a}_{\text {eq }}$ & $\left|H_{\text {ex }}\right|$ & $H_{\text {in }}$ & $\boldsymbol{I}_{\gamma}$ \\
\hline 1 & 40.9 & 5.4 & 3 & 505 & 22 & 930 & 285.39 & 39.95 & 505.18 & 1.64 & 3.01 & 4.21 \\
\hline 2 & 40.6 & 20.9 & 2.3 & 501 & 85 & 713 & 312.64 & 43.77 & 501.43 & 1.83 & 3.19 & 4.67 \\
\hline 3 & 40 & 18.1 & 4.9 & 494 & 73 & 1519 & 335.96 & 47.03 & 494.02 & 1.93 & 3.27 & 5.04 \\
\hline 4 & 40 & 6.2 & 2.8 & 494 & 25 & 868 & 279.63 & 39.15 & 494.06 & 1.61 & 2.95 & 4.12 \\
\hline 5 & 39.6 & 22.2 & 4.1 & 489 & 90 & 1271 & 333.39 & 46.67 & 489.08 & 1.93 & 3.26 & 5.01 \\
\hline 6 & 39.6 & 11 & 2.1 & 489 & 45 & 651 & 280.07 & 39.21 & 489.09 & 1.63 & 2.95 & 4.14 \\
\hline 7 & 39.5 & 21.3 & \begin{tabular}{|l|}
4.8 \\
\end{tabular} & 488 & 86 & 1488 & 339.66 & 47.55 & 487.84 & 1.96 & 3.28 & 5.11 \\
\hline 8 & 39.5 & 3.5 & 1.5 & 488 & 14 & 465 & 253.35 & 35.47 & 487.93 & 1.47 & 2.79 & 3.70 \\
\hline 9 & 39.5 & 6.2 & 2.8 & 488 & 25 & 868 & 276.77 & 38.75 & 487.88 & 1.60 & 2.91 & 4.08 \\
\hline 10 & 39.2 & 10 & 37 & 484 & 41 & 11470 & 726.48 & 101.71 & 484.16 & 3.85 & 5.16 & 11.28 \\
\hline 11 & 39.1 & 4 & 4 & 483 & 16 & 1240 & 284.61 & 39.85 & 482.97 & 1.63 & 2.93 & 4.21 \\
\hline 12 & 39.1 & 11.1 & 2.7 & 483 & 45 & 837 & 285.22 & 39.93 & 482.92 & 1.65 & 2.96 & 4.23 \\
\hline 13 & 39 & 4.5 & 1.6 & 482 & 18 & 496 & 254.24 & 35.59 & 481.73 & 1.48 & 2.78 & 3.72 \\
\hline 14 & 39 & 12.6 & 3.3 & 482 & 51 & 1023 & 08 & 45 & 481.68 & 1.71 & 3.01 & 4.40 \\
\hline 15 & 38.8 & 8.2 & 2 & 479 & 33 & 620 & 267.34 & 37.43 & 479.22 & 1.55 & 2.85 & 3.94 \\
\hline 16 & 38.5 & 24.5 & 4.9 & 475 & 99 & 1519 & 343.09 & 48.03 & 475.49 & 1.98 & 3.27 & 5.18 \\
\hline 17 & 38.4 & 3.1 & 2.2 & 474 & 13 & 682 & 255.14 & 35.72 & 474.35 & 1.47 & 2.75 & 3.74 \\
\hline 18 & 38.4 & 8.3 & 2.5 & 474 & 34 & 775 & 271.77 & 38.05 & 474.28 & 1.57 & 2.85 & 4.02 \\
\hline 19 & 38.3 & 17.8 & 3.1 & 473 & 72 & 961 & 5 & 42.32 & 473.02 & 1.76 & 3.04 & 4.52 \\
\hline 20 & 38.2 & 13.5 & \begin{tabular}{|l}
3.6 \\
\end{tabular} & 472 & 55 & 1116 & 297.60 & 41.66 & 471.80 & 1.72 & 2.99 & 4.44 \\
\hline 21 & 38.2 & 7.2 & 4.4 & 472 & 29 & 1364 & 292.49 & 40.95 & 471.82 & 1.67 & 2.95 & 4.35 \\
\hline 22 & 38.1 & 14 & 4.6 & 471 & 57 & 1426 & 311.18 & 43.57 & 470.56 & 1.79 & 3.06 & 4.66 \\
\hline 23 & 38.1 & 5 & 1.1 & 471 & 20 & 341 & 243.87 & 34.14 & 470.61 & 1.42 & 2.69 & 3.57 \\
\hline 24 & 37.9 & 3.6 & 1.1 & 468 & 15 & 341 & 239.29 & 33.50 & 468.16 & 1.39 & 2.66 & 3.49 \\
\hline 25 & 37.9 & 4.1 & 2.1 & 468 & 17 & 651 & 253.45 & 35.48 & 468.15 & 1.46 & 2.73 & 3.72 \\
\hline 26 & 37.8 & 3.1 & 0.9 & 467 & 13 & 279 & 234.91 & 32.89 & 466.94 & 1.37 & 2.63 & 3.42 \\
\hline 27 & 37.8 & 3.1 & 1.2 & 467 & 13 & 372 & 238.79 & 33.43 & 466.94 & 1.39 & 2.65 & 3.49 \\
\hline 28 & 37.7 & 10.3 & 2.3 & 466 & 42 & 713 & 270.10 & 37.81 & 465.63 & 1.57 & 2.83 & 4.00 \\
\hline 29 & 37.4 & 4.2 & 2.8 & 462 & 17 & 868 & 259.89 & 36.38 & 461.97 & 1.49 & 2.74 & 3.83 \\
\hline 30 & 37.3 & 5 & 3.4 & 461 & 20 & 1054 & 269.04 & 37.66 & 460.73 & 1.54 & 2.79 & 3.98 \\
\hline Average & 38.78 & 9.73 & 3.97 & 478.93 & 39.52 & 1230.70 & 296.46 & 41.50 & 478.99 & 1.70 & 3.00 & 4.41 \\
\hline
\end{tabular}


Table (3): Environmental radioactivity parameters of the altered zone.

\begin{tabular}{|c|c|c|c|c|c|c|c|c|c|c|c|c|}
\hline $\begin{array}{c}\text { Sample } \\
\text { No. }\end{array}$ & $\begin{array}{c}\mathrm{eU} \\
(\mathrm{ppm})\end{array}$ & $\begin{array}{c}\text { eTh } \\
(\mathbf{p p m})\end{array}$ & $\begin{array}{c}\mathrm{K} \\
(\%)\end{array}$ & $\begin{array}{c}\mathrm{eU} \\
\mathrm{Bq} / \mathrm{kg}\end{array}$ & $\begin{array}{c}\text { eTh } \\
\text { Bq/kg }\end{array}$ & $\begin{array}{c}\mathrm{K} \\
\mathrm{Bq} / \mathrm{kg}\end{array}$ & $\begin{array}{c}\text { Abs. Dose } \\
{ }^{238} \mathrm{U}(\mathrm{nGy} / \mathrm{h})\end{array}$ & $\begin{array}{c}\text { Eff. Dose } \\
(\mathrm{mSv})\end{array}$ & $\mathbf{R a}_{\text {eq }}$ & $H_{\text {ex }}$ & $\boldsymbol{H}_{\text {in }}$ & $I_{\gamma}$ \\
\hline 1 & 365 & 259 & 2.3 & 4508 & 1052 & 713 & .44 & 384.64 & 4507.75 & 16.39 & 28.57 & 41.04 \\
\hline 2 & 364.3 & 315 & 5.4 & 4499 & 1279 & 1674 & 2920.85 & 408.92 & 4499.11 & 17.45 & 29.61 & 43.90 \\
\hline 3 & 358 & 304 & 2.4 & 4421 & 1234 & 744 & 2819.15 & 394.68 & 4421.30 & 16.87 & 28.82 & 42.31 \\
\hline 4 & 317 & 313 & 3.2 & 3915 & 1271 & 992 & 2617.62 & 366.47 & 3914.95 & 15.69 & 26.27 & 39.47 \\
\hline 5 & 315 & 314 & 5.4 & 3890 & 1275 & 1674 & & 369.19 & 3890.25 & 15.78 & 26.30 & 39.80 \\
\hline 6 & 295.7 & 319 & 5.8 & 3652 & 1295 & 1798 & 2544.42 & 356.22 & 3651.90 & 15.24 & 25.11 & 38.50 \\
\hline 7 & 288 & 313 & 3.4 & 3557 & 1271 & 1054 & 2454.74 & 343.66 & 3556.80 & 14.74 & 24.35 & 37.12 \\
\hline 8 & 288 & 310 & 2.8 & 3557 & 1259 & 868 & 2439.63 & 341.55 & 3556.80 & 14.65 & 24.27 & 36.88 \\
\hline 9 & 285.3 & 209 & 4.5 & 3523 & 849 & 1395 & 2198.53 & 307.79 & 3523.46 & 13.09 & 22.61 & 32.91 \\
\hline 10 & 285.3 & 216 & 4.5 & 3523 & 877 & 1395 & 2215.69 & 310.20 & 3523.46 & 13.20 & 22.72 & 33.19 \\
\hline 11 & 279 & 311 & 4 & 3446 & 1263 & 1240 & 2406.24 & 336.87 & 3445.65 & 14.45 & 23.76 & 36.42 \\
\hline 12 & 276 & 321 & 3.8 & 3409 & 1303 & 1178 & 2411.06 & 337.55 & 3408.60 & 14.49 & 23.70 & 36.54 \\
\hline 13 & 265.9 & 306 & 3.9 & 3284 & 1242 & 1209 & 2317.95 & 324.51 & 3283.87 & 13.92 & 22.80 & 35.12 \\
\hline 14 & 253.5 & 312 & 5.4 & 3131 & 1267 & 1674 & 2281.30 & 319.38 & 3130.73 & 13.70 & 22.16 & 34.65 \\
\hline 15 & 242.9 & 295 & 3.9 & 3000 & 1198 & 1209 & 9.74 & .36 & 2999.82 & 12.98 & 21.09 & 32.78 \\
\hline 16 & 242 & 313 & 4.6 & 2989 & 1271 & 1426 & 2207.79 & 309.09 & 2988.70 & 13.28 & 21.36 & 33.58 \\
\hline 17 & 239.2 & 311 & 6.1 & 2954 & 1263 & 1891 & 2206.30 & 308.88 & 2954.12 & 13.25 & 21.24 & 33.58 \\
\hline 18 & 237.4 & 322 & 4.8 & 2932 & 1307 & 1488 & 2206.20 & 308.87 & 2931.89 & 13.28 & 21.20 & 33.61 \\
\hline 19 & 237.3 & 309 & 4.1 & 2931 & 1255 & 1271 & 2164.71 & 303.06 & 2930.66 & 13.03 & 20.95 & 32.93 \\
\hline 20 & 233.6 & 307 & 3.9 & 2885 & 1246 & 1209 & 2136.10 & 299.05 & 2884.96 & 12.86 & 20.66 & 32.50 \\
\hline 21 & 229.5 & 308 & 4.4 & 2834 & 1250 & 1364 & 2121.63 & 297.03 & 2834.33 & 12.77 & 20.43 & 32.31 \\
\hline 22 & 215.3 & 315 & 5.5 & 2659 & 1279 & 1705 & 2071.99 & 290.08 & 2658.96 & 12.48 & 19.67 & 31.65 \\
\hline 23 & 211.8 & 306 & 5.1 & 2616 & 1242 & 1581 & 2024.78 & 283.47 & 2615.73 & 12.19 & 19.26 & 30.92 \\
\hline 24 & 206 & 305 & 1.7 & 2544 & 1238 & 527 & 1945.28 & 272.34 & 2544.10 & 11.77 & 18.64 & 29.70 \\
\hline 25 & 206 & 308 & 5.5 & 2544 & 1250 & 1705 & 2001.76 & 280.25 & 2544.10 & 12.06 & 18.93 & 30.60 \\
\hline 26 & 205 & 306 & 3.1 & 2532 & 1242 & 961 & 1960.13 & 274.42 & 2531.75 & 11.84 & 18.68 & 29.94 \\
\hline 27 & 205 & 259 & 5.5 & 2532 & 1052 & 1705 & .90 & .63 & 2531.75 & 11.26 & 18.10 & 28.53 \\
\hline 28 & 204.7 & 311 & 4.6 & 2528 & 1263 & 1426 & 1990.07 & 278.61 & 2528.05 & 12.00 & 18.84 & 30.43 \\
\hline 29 & 203.8 & 317 & 3.8 & 2517 & 1287 & 1178 & 1989.30 & 278.50 & 2516.93 & 12.02 & 18.82 & 30.44 \\
\hline 30 & 203.7 & 314 & 5 & 2516 & 1275 & 1550 & 1996.89 & 279.56 & 2515.70 & 12.04 & 18.84 & 30.55 \\
\hline Average & 258.64 & 300.93 & 4.28 & 3194.20 & 1221.79 & 1326.80 & 2269.01 & 317.66 & 3194.21 & 13.63 & 22.26 & 34.40 \\
\hline
\end{tabular}


Table (4): Environmental radioactivity parameters of the inner quartz zone.

\begin{tabular}{|c|c|c|c|c|c|c|c|c|c|c|c|c|}
\hline $\begin{array}{c}\text { Sample } \\
\text { No. }\end{array}$ & $\begin{array}{c}\mathrm{eU} \\
(\mathrm{ppm})\end{array}$ & $\begin{array}{c}\text { eTh } \\
\text { (ppm) }\end{array}$ & \begin{tabular}{|c|}
$\mathbf{K}$ \\
$(\%)$ \\
\end{tabular} & \begin{tabular}{c|}
$\mathrm{eU}$ \\
$\mathrm{Bq} / \mathrm{kg}$
\end{tabular} & \begin{tabular}{|c|} 
eTh \\
Bq/kg \\
\end{tabular} & $\begin{array}{c}\mathrm{K} \\
\mathrm{Bq} / \mathrm{kg}\end{array}$ & $\begin{array}{c}\text { Abs. Dose } \\
{ }^{238} \mathbf{U}(\mathbf{n G y} / \mathbf{h})\end{array}$ & $\begin{array}{c}\text { Eff. Dose } \\
(\mathrm{mSv})\end{array}$ & $\mathbf{R a}_{\text {eq }}$ & $H_{\text {ex }}$ & $H_{\text {in }}$ & $\boldsymbol{I}_{\gamma}$ \\
\hline 1 & 24 & 9 & 1.7 & 296 & 37 & 527 & 180.98 & 25.34 & 296.44 & 1.05 & 1.85 & 2.69 \\
\hline 2 & 23.9 & 9 & 4.7 & 295 & 37 & 1457 & 219.19 & 30.69 & 295.20 & 1.24 & 2.04 & 3.30 \\
\hline 3 & 23.9 & 9 & 3.7 & 295 & 37 & 1147 & 206.27 & 28.88 & 295.20 & 1.18 & 1.98 & 3.10 \\
\hline 4 & 23.9 & 8 & 2.6 & 295 & 32 & 806 & 189.59 & 26.54 & 295.21 & 1.09 & 1.89 & 2.83 \\
\hline 5 & 23.8 & 8 & 2.7 & 294 & 32 & 837 & 190.32 & 26.64 & 293.97 & 1.09 & 1.89 & 2.84 \\
\hline 6 & 23.8 & 10 & 3.8 & 294 & 41 & 1178 & 209.44 & 29.32 & 293.97 & 1.20 & 1.99 & 3.15 \\
\hline 7 & 23.8 & 10 & 3.3 & 294 & 41 & 1023 & 202.98 & 28.42 & 293.97 & 1.16 & 1.96 & 3.05 \\
\hline 8 & 23.8 & 10 & 3.5 & 294 & 41 & 1085 & 205.56 & 28.78 & 293.97 & 1.18 & 1.97 & 3.09 \\
\hline 9 & 23.8 & 10 & 3.5 & 294 & 41 & 1085 & 205.56 & 28.78 & 293.97 & 1.18 & 1.97 & 3.09 \\
\hline 10 & 23.8 & 10 & 44 & 294 & 41 & 13640 & 729.11 & 102.07 & 293.97 & 3.79 & 4.58 & 11.46 \\
\hline 11 & 23.7 & 10 & 4.2 & 293 & 41 & 1302 & 214.04 & 29.97 & 292.73 & 1.22 & 2.01 & 3.23 \\
\hline 12 & 23.7 & 10 & 3 & 293 & 41 & 930 & 198.53 & 27.79 & 292.73 & 1.14 & 1.93 & 2.98 \\
\hline 13 & 23.7 & 10 & 7.1 & 293 & 41 & 2201 & 251.53 & 35.21 & 292.73 & 1.41 & 2.20 & 3.82 \\
\hline 14 & 23.6 & 10 & 4 & 291 & 41 & 1426 & & 61 & 291.50 & 1.24 & 2.03 & 3.30 \\
\hline 15 & 23.6 & 10 & 3.8 & 291 & 41 & 1178 & 208.30 & 29.16 & 291.50 & 1.19 & 1.98 & 3.13 \\
\hline 16 & 23.6 & 11 & 2.8 & 291 & 45 & 868 & 197.82 & 27.70 & 291.49 & 1.14 & 1.93 & 2.97 \\
\hline 17 & 23.6 & 11 & 6.4 & 291 & 45 & 1984 & 244.36 & 34.21 & 291.49 & 1.37 & 2.16 & 3.71 \\
\hline 18 & 23.5 & 11 & 5 & 290 & 45 & 1550 & 225.69 & 31.60 & 290.26 & 1.28 & 2.06 & 3.41 \\
\hline 19 & 23.5 & 11 & 5.1 & 290 & 45 & 1581 & 99 & 31.78 & 290.26 & 1.29 & 2.07 & 3.44 \\
\hline 20 & 23.5 & 11 & 2.9 & 290 & 45 & 899 & 198.55 & 27.80 & 290.26 & 1.14 & 1.93 & 2.98 \\
\hline 21 & 23.5 & 11 & 3.1 & 290 & 45 & 961 & 201.13 & 28.16 & 290.26 & 1.16 & 1.94 & 3.02 \\
\hline 22 & 23.5 & 11 & 2.6 & 290 & 45 & 806 & 194.67 & 27.25 & 290.26 & 1.12 & 1.91 & 2.92 \\
\hline 23 & 23.4 & 11 & 4.1 & 289 & 45 & 1271 & 213.49 & 29.89 & 289.02 & 1.22 & 2.00 & 3.22 \\
\hline 24 & \begin{tabular}{|l|}
23.4 \\
\end{tabular} & 11 & 3.9 & 289 & 45 & 1209 & 210.90 & 29.53 & 289.02 & 1.20 & 1.99 & 3.18 \\
\hline 25 & 23.3 & 11 & 1.5 & 288 & 45 & 465 & 179.31 & 25.10 & 287.79 & 1.05 & 1.82 & 2.67 \\
\hline 26 & 23.3 & 11 & 1.5 & 288 & 45 & 465 & 179.31 & 25.10 & 287.79 & 1.05 & 1.82 & 2.67 \\
\hline 27 & 23.3 & 11 & 5.9 & 288 & 45 & 1829 & 236.19 & 33.07 & 287.79 & 1.33 & 2.11 & 3.58 \\
\hline 28 & 23.3 & 11 & 2.9 & 288 & 45 & 899 & 197.41 & 27.64 & 287.79 & 1.14 & 1.91 & 2.96 \\
\hline 29 & 23.2 & 11 & 2 & 287 & 45 & 620 & 185.20 & 25.93 & 286.55 & 1.08 & 1.85 & 2.77 \\
\hline 30 & 23.1 & 11 & 4 & 285 & 45 & 1240 & 210.48 & 29.47 & 285.32 & 1.20 & 1.97 & 3.18 \\
\hline Average & 23.59 & 10.27 & 5.00 & 291.38 & 41.68 & 1548.97 & 224.38 & 31.41 & 291.41 & 1.27 & 2.06 & 3.39 \\
\hline
\end{tabular}

- Calculate the uranium mobilization rate $(\mathrm{P})$

$$
\mathrm{P}=\mathrm{Um} / \mathrm{Up} * 100 \%
$$

The obtained results of Uo, Um and P for the studied pegmatite zones are listed in Table (5). The amount of mobilized (migrated) uranium (Um) is calculated by $\mathrm{Um}=\mathrm{Up}-\mathrm{Uo}$, where, $\mathrm{Um}$ is the amount of the mobilized uranium and Up is the average 
of the present uranium content in certain geologic unit. The calculated values of the mobilized uranium (Um) indicate that uranium was mobilized, with different trends and amounts, through all the studied pegmatite zones and related rocks. The Um values are $>0$ in the outer, intermediate and inner pegmatite zones but $<0$ in the altered zone. This indicates mobilization of $U$ into these zones (migration in) and out of the altered zone (migration out). This indicates that uranium migrates from the altered pegmatite zone to the other zones.

Table (5): The original uranium, the present uranium, the mobilized uranium and the uranium mobilization rate in the studied pegmatite zones.

\begin{tabular}{|l|c|c|c|c|}
\hline & Uo $(\mathbf{p p m})$ & $\mathbf{U p}(\mathbf{p p m})$ & $\mathbf{U m}(\mathbf{p p m})$ & $\mathbf{P} \%$ \\
\hline Outer zone & 53.97 & 63.01 & 9.04 & 14.34 \\
\hline Intermed zone & 9.51 & 38.78 & 29.27 & 75.49 \\
\hline Altered zone & 293.88 & 258.64 & -35.24 & -13.63 \\
\hline Inner qz zone & 10.03 & 23.59 & 13.57 & 57.50 \\
\hline
\end{tabular}

The eU-eTh, (eU/eTh)-eU, (eU/eTh)-eTh and eU-[eU+(eTh*3.5)] diagrams for the studied pegmatite zones (Fig. 3a, b \&d) show enrichment of uranium for the outer, intermediate and inner zones of the studied pegmatite. The altered zone (Fig. 3c) shows uranium depletion suggesting that uranium was leached out of the high radioactive altered zone towards the outer, intermediate and inner pegmatite zones.

\section{MINERALOGY}

The accessory minerals of the alteration zone of the mineralized pegmatite were identified by XRD and ESEM techniques. They could be categorized into three groups:

a- Uranium minerals: The examination of hand-picked radioactive grains from the mineralized pegmatite using X- ray Diffraction are shown in (Tables 6 and 7). The Environmental Scanning Electron Microscope (Figs. 3a and 3b) confirms the presence of betafite and kasolite as the principal secondary uranium minerals.

b- Radioactive accessory minerals such as zircon (Fig. 4a) and fluorite (Fig. 4b).

c- Non-radioactive accessory minerals such as calcite, kaolinite, pyrite, hematite, rutile and columbite (Fig. 4c). 


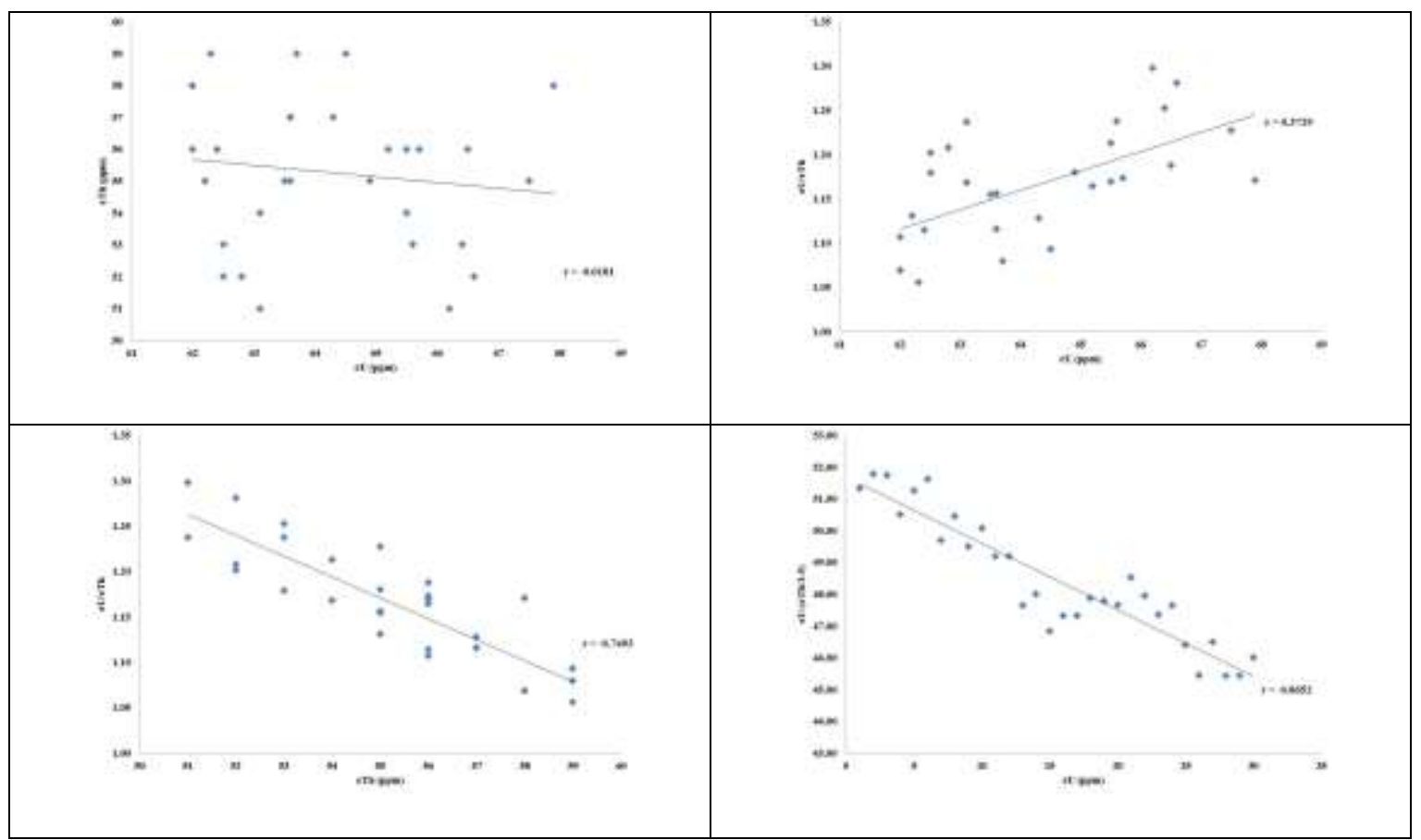

Fig. (3a): The eU-eTh, (eU/eTh)-eU, (eU/eTh)-eTh and eU-[eU+(eTh*3.5)] diagrams for the outer feldspar zone.

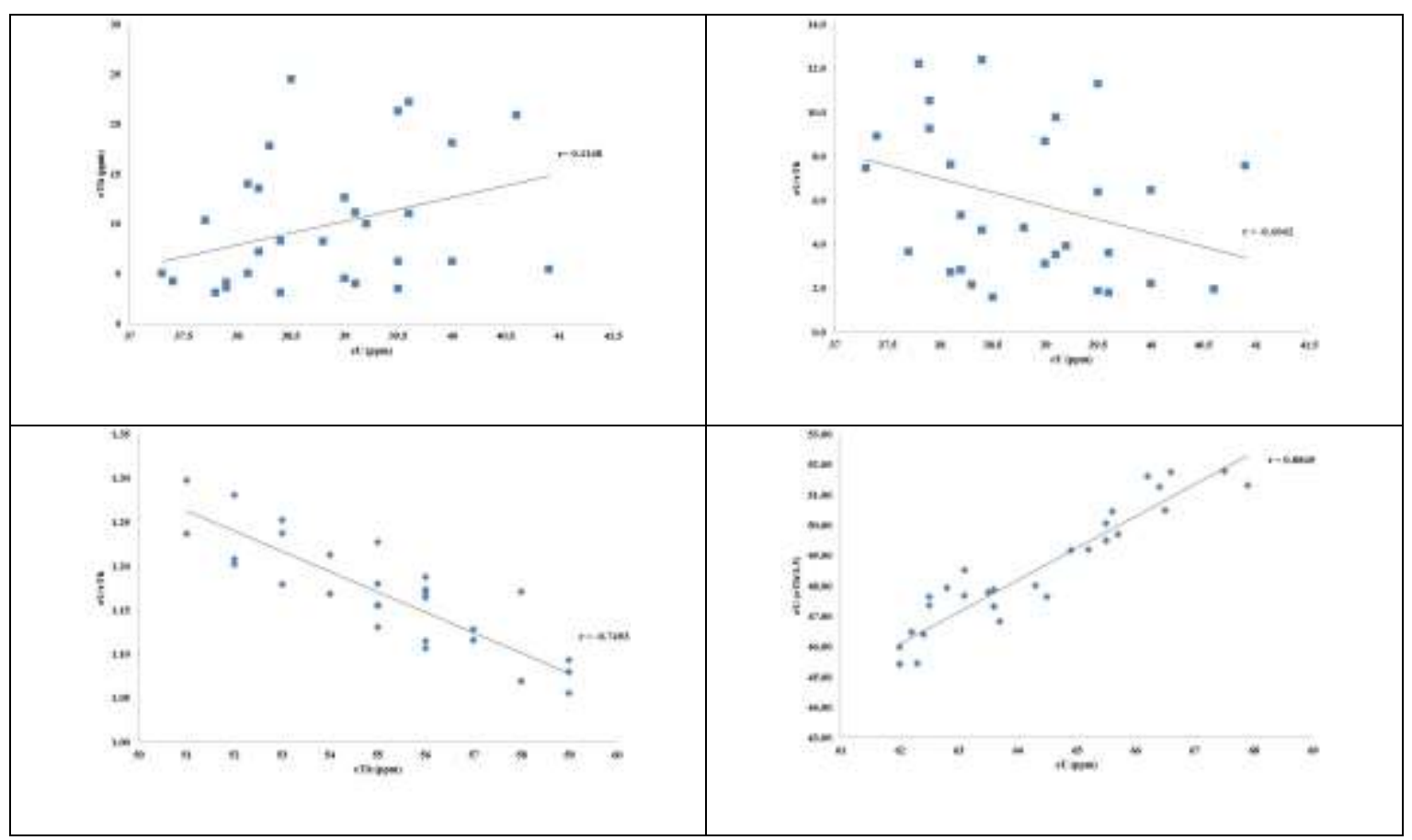

Fig. (3b): The eU-eTh, (eU/eTh)-eU, (eU/eTh)-eTh and eU-[eU+(eTh*3.5)] diagrams for the intermediate mica zone. 


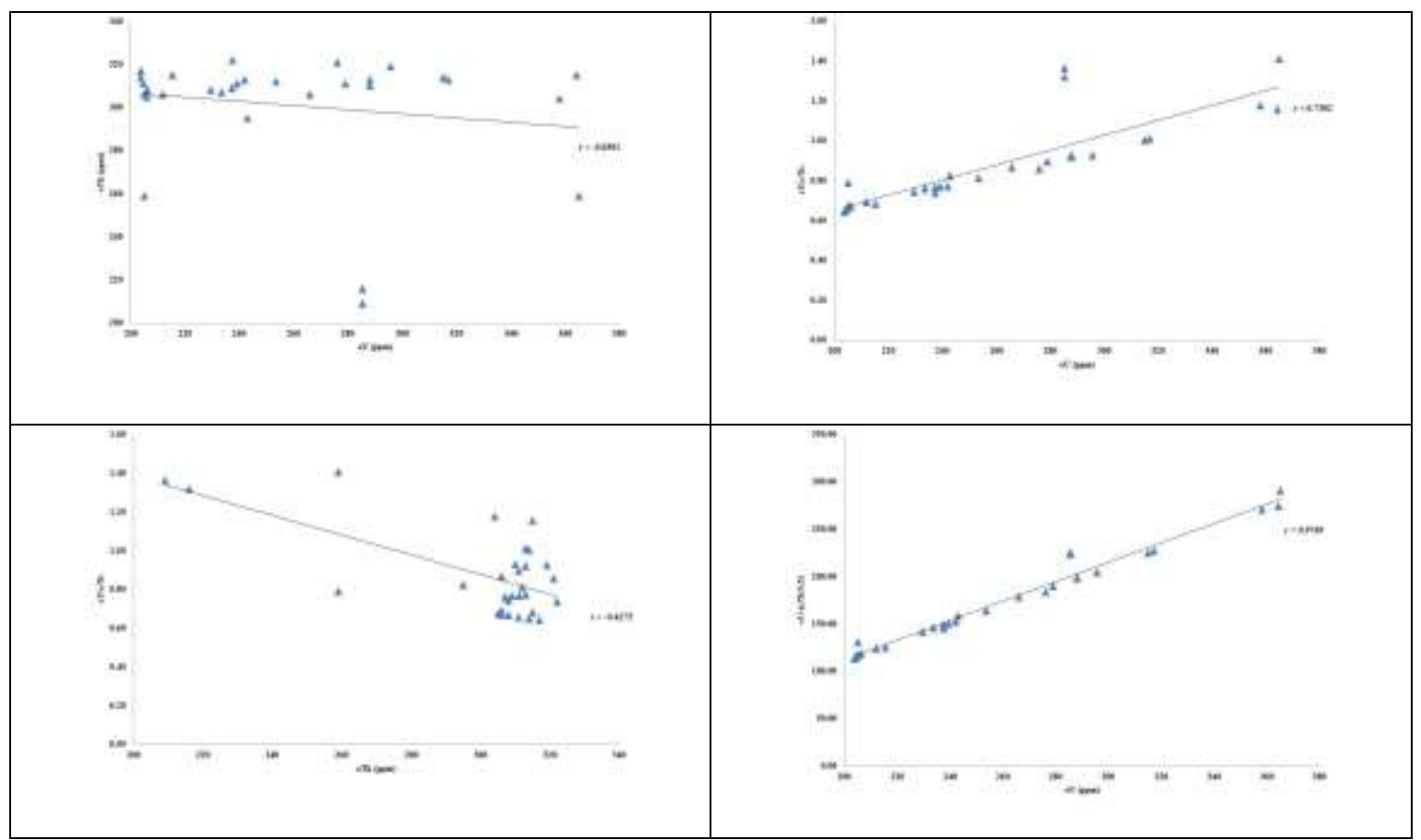

Fig. (3c): The eU-eTh, (eU/eTh)-eU, (eU/eTh)-eTh and eU-[eU+(eTh*3.5)] diagrams for the altered zone.

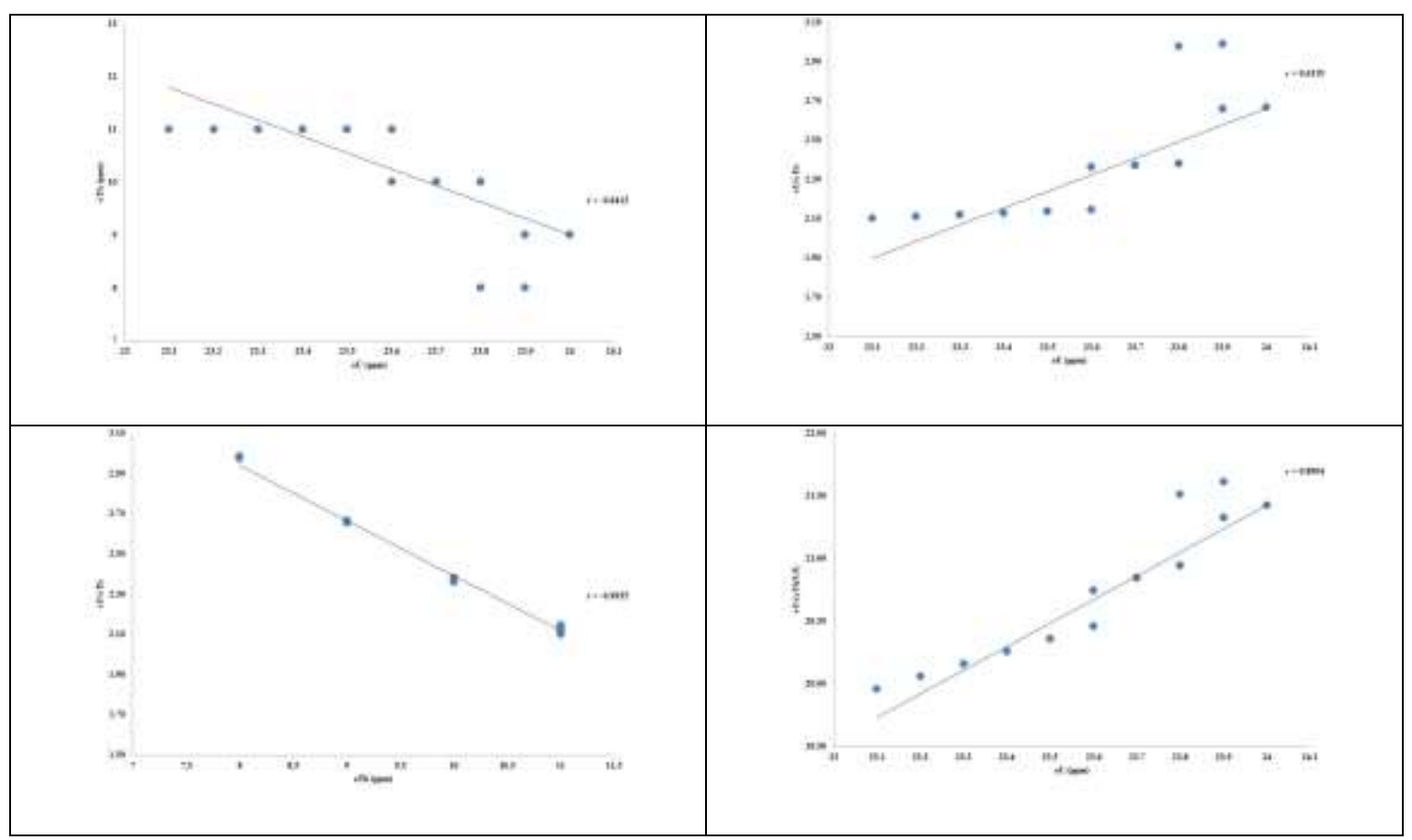

Fig. (3d): The eU-eTh, (eU/eTh)-eU, (eU/eTh)-eTh and eU-[eU+(eTh*3.5)] diagrams for the inner quartz zone. 
Table (6): X-ray diffraction pattern of hand picked betafite mineral samples.

\begin{tabular}{|c|c|c|c|c|c|}
\hline \multicolumn{2}{|c|}{ Sample No. 15} & \multicolumn{2}{|c|}{ Sample No. 18} & \multicolumn{2}{|c|}{ Betafite * } \\
\hline $\mathrm{dA}^{\mathrm{o}}$ & $\mathrm{I} / \mathrm{I}_{\mathrm{O}}$ & $\mathrm{dA}^{\mathrm{o}}$ & $\mathrm{I} / \mathrm{I}_{\mathrm{O}}$ & $\mathrm{dA}^{\mathrm{o}}$ & $\mathrm{I} / \mathrm{I}_{\mathrm{O}}$ \\
\hline 2.94 & 100 & 2.96 & 100 & 2.96 & 100 \\
\hline 2.50 & 20 & 2.61 & 25 & 2.56 & 20 \\
\hline 2.00 & 5 & 1.94 & 3 & 1.97 & 3 \\
\hline 1.87 & 45 & 1.85 & 45 & 1.81 & 45 \\
\hline 1.54 & 40 & 1.54 & 35 & 1.54 & 40 \\
\hline 1.48 & 15 & 1.48 & 15 & 1.48 & 15 \\
\hline 1.28 & 10 & 1.28 & 10 & 1.28 & 10 \\
\hline 1.18 & 18 & 1.18 & 20 & 1.18 & 20 \\
\hline 1.15 & 15 & 1.24 & 15 & 1.15 & 15 \\
\hline 1.65 & 60 & 1.62 & 55 & 1.65 & 60 \\
\hline 1.50 & 10 & 1.41 & 12 & 1.48 & 10 \\
\hline
\end{tabular}

* ASTM card No. 8-300
Table (7): X-ray diffraction pattern of hand picked kasolite mineral samples.

\begin{tabular}{||c|c|c|c|c|c||}
\hline \multicolumn{2}{||c|}{ Sample No. 15 } & \multicolumn{2}{|c|}{ Sample No. 18 } & \multicolumn{2}{c|}{ Kasolite*** $^{* *} \mathrm{I}^{\mathrm{o}}$} \\
\hline $\mathrm{dA}^{\mathrm{o}}$ & $\mathrm{I} / \mathrm{I}_{\mathrm{O}}$ & $\mathrm{dA}^{\mathrm{o}}$ & $\mathrm{I} / \mathrm{I}_{\mathrm{O}}$ & $\mathrm{dA}^{\mathrm{O}}$ & $\mathrm{I}^{\mathrm{I}} \mathrm{O}$ \\
\hline 2.95 & 100 & 2.90 & 100 & 2.92 & 100 \\
6.14 & 40 & 6.10 & 35 & 6.15 & 40 \\
-- & 5 & 4.90 & 5 & 4.73 & 5 \\
4.30 & 65 & 4.32 & 55 & 4.21 & 60 \\
3.31 & 55 & 3.26 & 60 & 3.24 & 60 \\
2.98 & 80 & 3.11 & 75 & 3.06 & 80 \\
2.60 & 5 & 2.57 & 5 & 2.65 & 5 \\
2.12 & 15 & 2.05 & 25 & 2.18 & 20 \\
2.12 & 5 & 2.00 & 5 & 2.04 & 5 \\
2.00 & 5 & -- & 5 & 1.96 & 5 \\
1.88 & 15 & 1.85 & 20 & 1.87 & 20 \\
\hline
\end{tabular}

** ASTM card No. 12-210

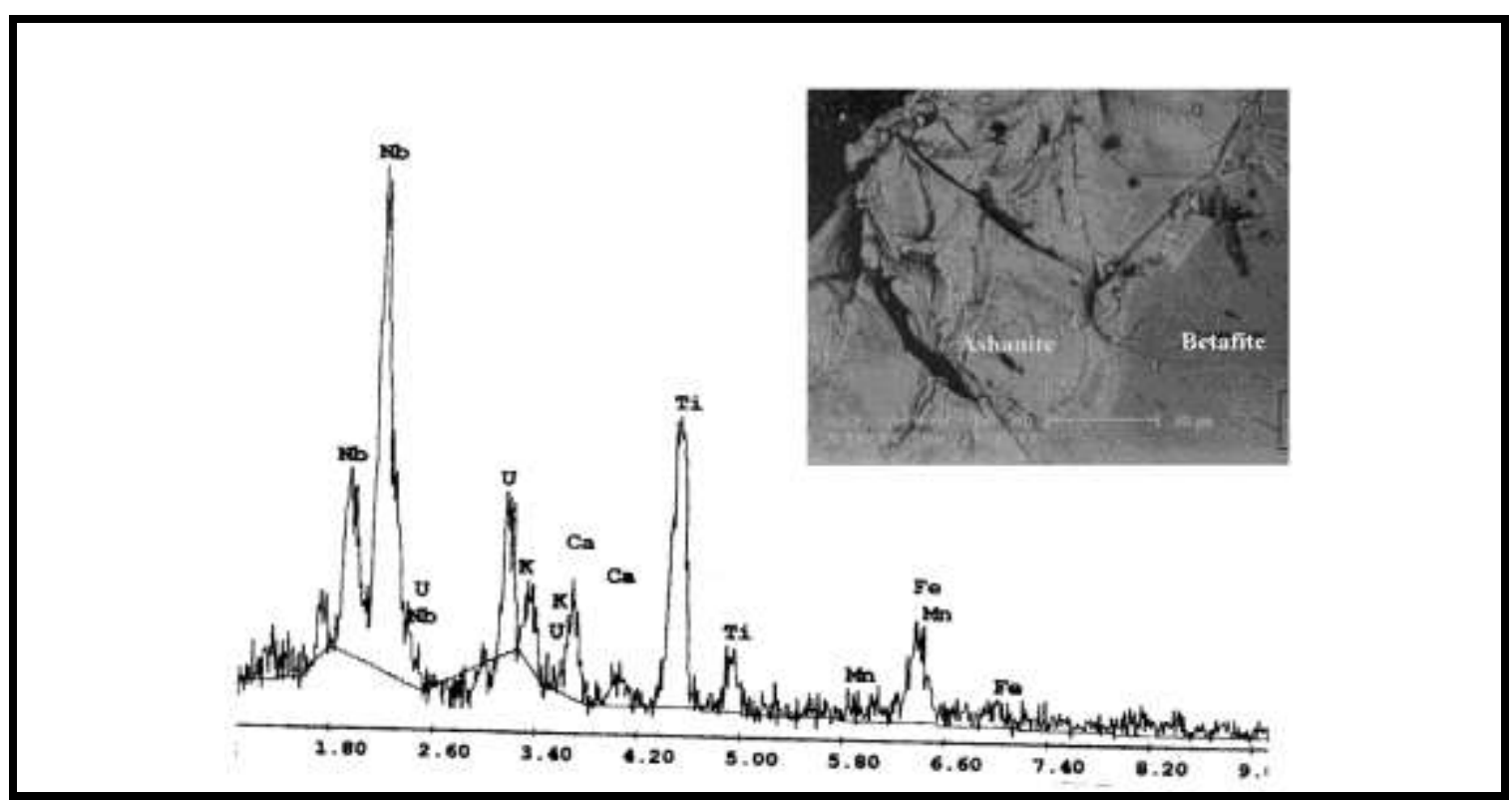

Fig. (3a): EDX spectrum and BSE image of betafite.

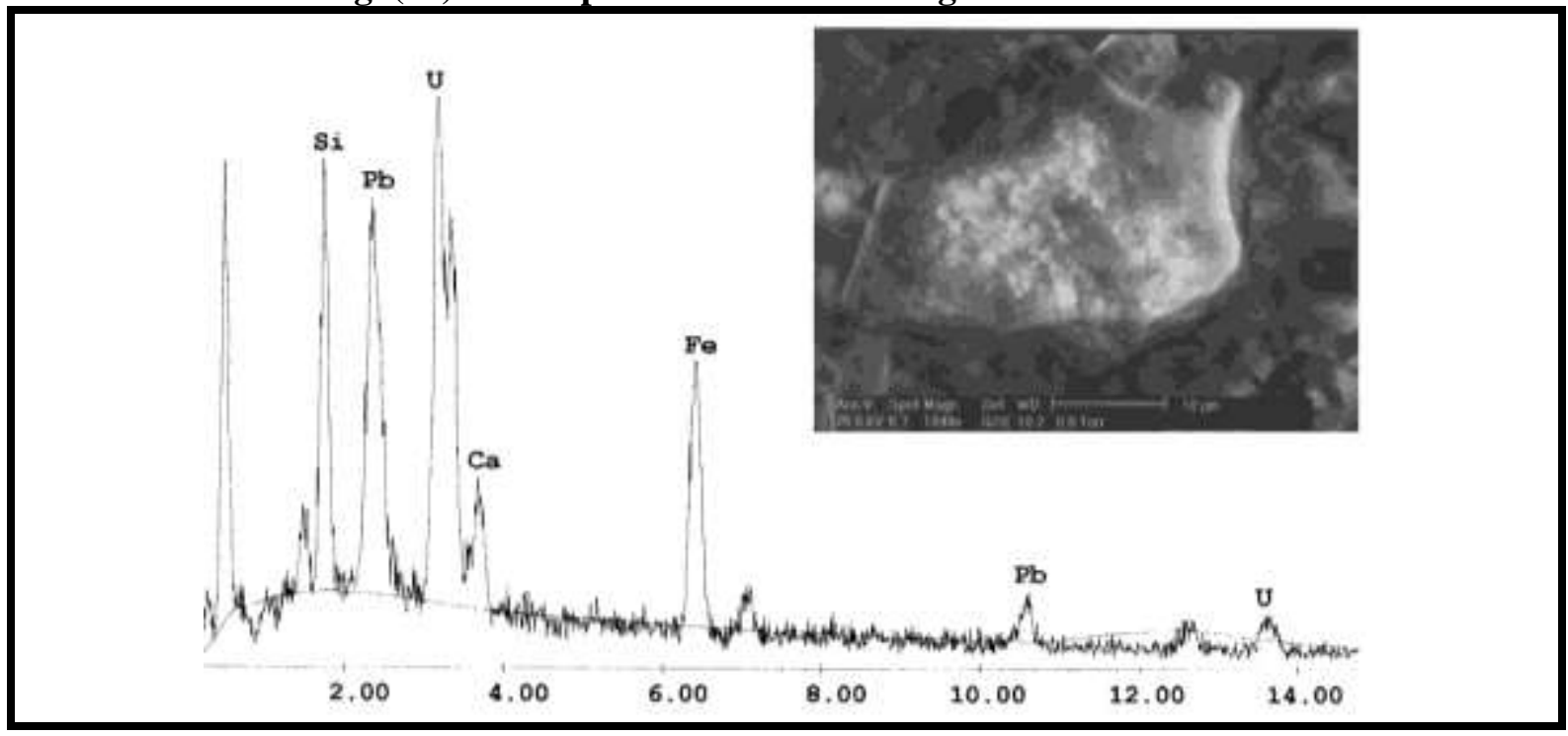

Fig. (3b): EDX spectrum and BSE image of kasolite. 


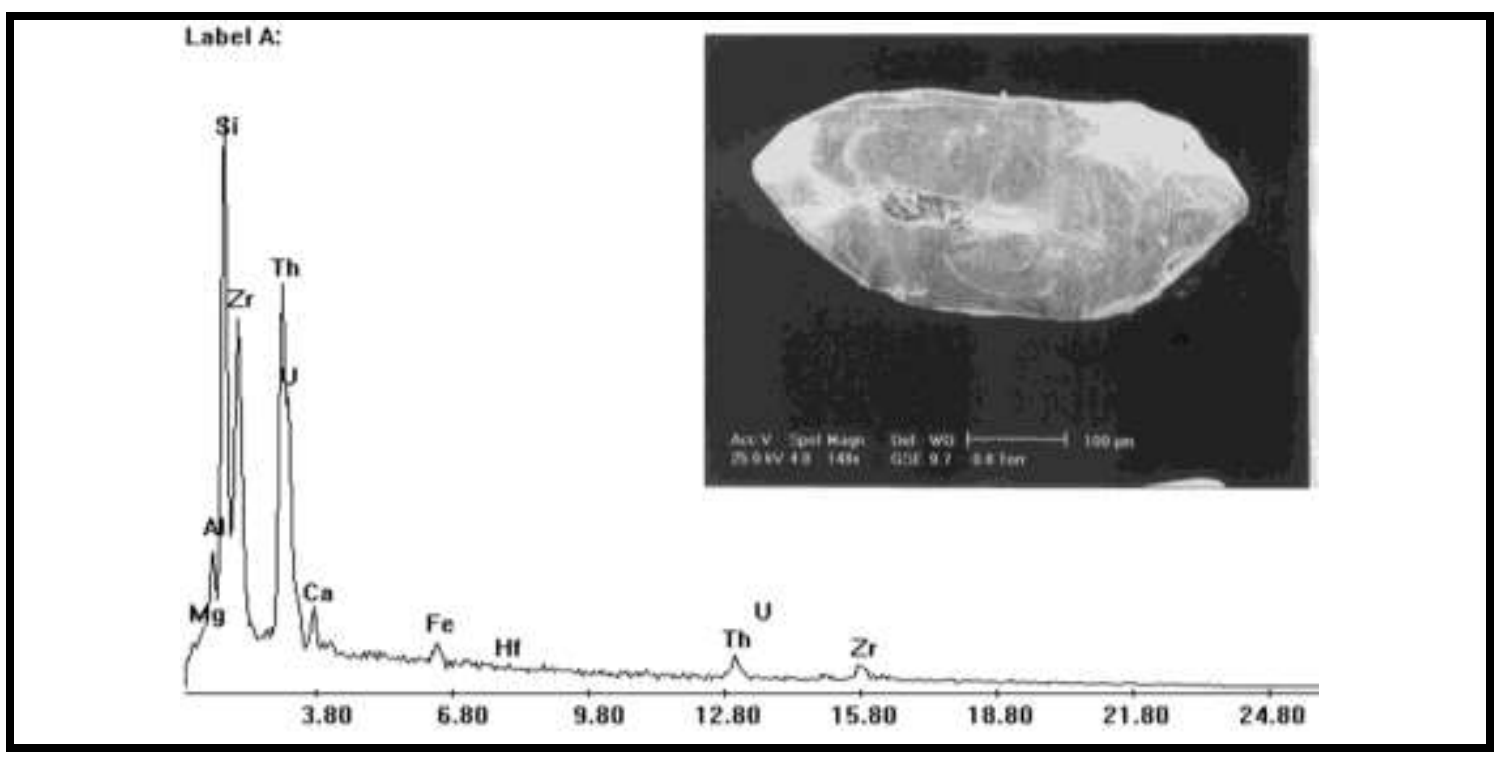

Fig. (4a): EDX spectrum and BSE image of zircon and thorite.

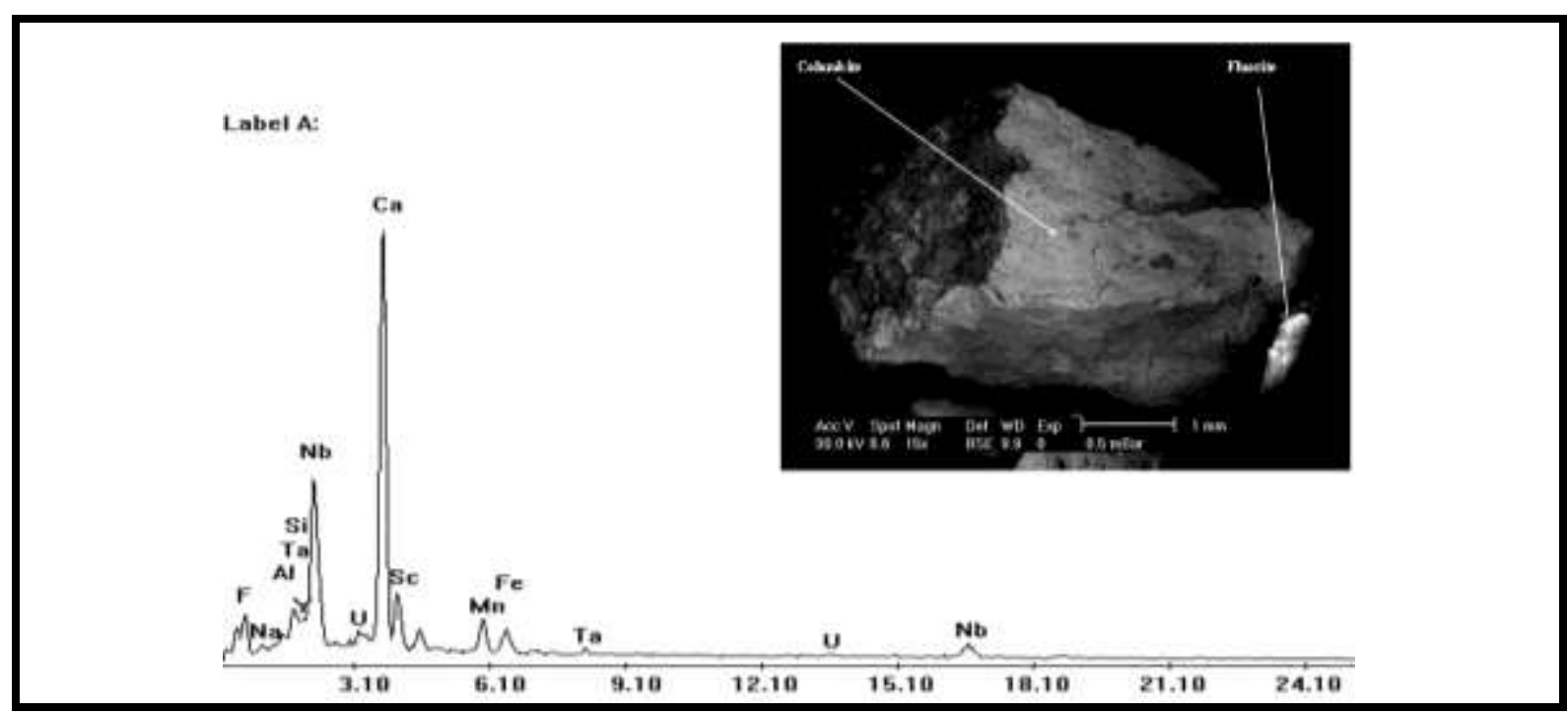

Fig. (4b): EDX pectrum and BSE image of fluorite in columbite. 


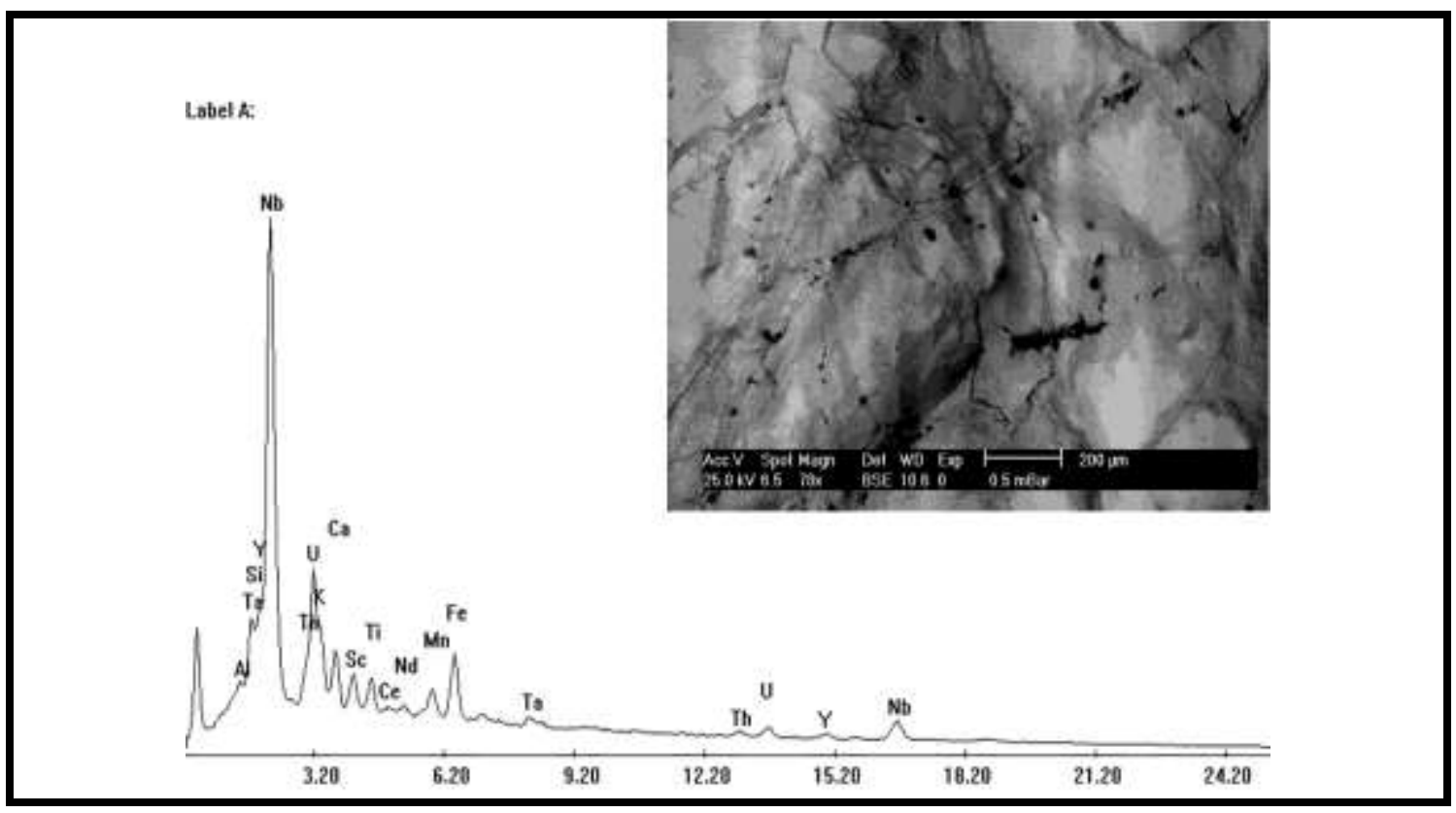

Fig. (4c): EDX spectrum and BSE image of columbite.

Berry, et al. (2000) stated that U-bearing columbite is Ta-poor and lesser amounts of $U$ could be accommodated in the columbite structure with decreasing temperature of crystallization. Rismaite (1977) stated that, kasolite ranks second in abundance after uranophane (and beta-uranophane). It occurs in pegmatite and formed under oxidizing conditions. Maurice (1982) suggested that kasolite is dimorphous with uranophane and beta-uranophane due to the complete replacement of $\mathrm{Ca}$ by $\mathrm{Pb}$ during the high degree of oxidation condition.

Babu (1969) applied several geothermometers to the pegmatite of India and reported temperature of $700^{\circ} \mathrm{C}$ for the pegmatitic magma with descending temperature of $670^{\circ}$ to $500^{\circ} \mathrm{C}$ for biotite, $600^{\circ}$ to $300^{\circ} \mathrm{C}$ for feldspars, $500^{\circ}$ to $435^{\circ} \mathrm{C}$ for muscovite, $500^{\circ}$ to $400^{\circ} \mathrm{C}$ for milky quartz and $300^{\circ} \mathrm{C}$ for clear quartz in the core.

\section{SUMMARY AND CONCLUSIONS}

From the obtained results, it is noticed that Gabal Ras Baroud zoned pegmatite mineral resources are characterized by the following:

1- From a paragenetic point of view, zones in pegmatite are developed from the wall inward within a restricted pegmatitic stages. In the early stage, zoned pegmatites of simple mineralogy are produced. In the later stage, residual fluids rich in soda, silica, alumina and significant quantities of $\mathrm{Nb}, \mathrm{Ta}, \mathrm{U}$, Th and $\mathrm{F}$ travel along fractures, react with the previously formed pegmatitic minerals forming new minerals of replacement origin. During this hydrothermal stage that most of the secondary uranium minerals, muscovite and columbite were formed (Chapman and Hall, 1997). In Ras Baroud pegmatites, the occurrence of uranium mineralization and columbite within the feldspar zone $\left(600^{\circ}-300^{\circ} \mathrm{C}\right)$ is in close association with muscovite $(500$ $435^{\circ} \mathrm{C}$ ), but at the contact with the quartz core falling to $300^{\circ} \mathrm{C}$, which confines their 
formation in the range between $500-300^{\circ} \mathrm{C}$ (Op.cit). This range of temperature is further confirmed by the presence of minerals in the low temperature polymorphic series.

2- The altered zone of pegmatite exhibit the highest radioactive values due to their mineral composition, as a result of alteration processes associated with columbite, radioactive accessory minerals and U-minerals. The alteration is mainly due to volatile-rich hydrothermal solutions that evolved from late differentiated magmatic fluids (Chapman and Hall, 1997).

3- The calculated environmental radioactivity parameters for the four zones of the pegmatite reflect hazardous effects on humans since they exceed the safety limit.

4- The calculated paleo- uranium content, uranium migration in and/or out as well as uranium migration rate showed that uranium migrates in the outer, intermediate and inner pegmatite zones while migrates out of the high radioactive altered zone which makes these zones harmful for industrial use as building or ornamental stones. They can be used only in outdoor to avoid radon gas accumulation. Also, they have to be stored in well ventilated warehouses and handled with care.

5- The accessory minerals (fluorite, zircon and hematite) concentrate much more $U$ than Th, due to the relative less mobility of Th than $U$, supporting the hydrothermal concept of U enrichment (Cathelineau and Holliger, 1987).

\section{REFERENCES}

Abbady, A. G., Uosif, M. A. and El-Taher, A. (2005): Natural radioactivity and dose assessment for phosphate rocks from Wadi El-Mashash and El-Mahamid Mines, Egypt. Journal of Environmental Radioactivity 84(1):65-78.

Abdel Monsif, M., El Nahas, H. and Abdallah, S. (2018): Mineralogy and trace elements geochemistry of pegmatite body at the northern periphery of Gabal Ras Baroud, Central Eastern Desert, Egypt. Nuclear Sciences Scientific Journal, 7, $151-164$.

Abd El Warith, A. (2000): Geology, geochemistry and radioactivity of the granitoid rocks in Gabal El-Magal area, central Eastern Desert, Egypt. Ph. D. Thesis, Faculty of Science, Ain Shams University, Cairo, Egypt, 208 p.

Assaf, H. S., Mahdy, M. A. and El Afandy, A. H. (1997): Egyptian younger granites, an approach to define parameters favouring formation of uranium deposits. $3^{\text {rd }}$ Conference Geochemistry, Alexandria Univ., Egypt. p. 409 - 420.

Babu. V. R. (1969): Temperature of formation of pegmatites of the Nellore mica belt, Andra Pradesh, India, Econ. Geol., V. 64, p. 66-71. 
Baykara, O., Karatepe, S. and Do־gru, M. (2010): Assessments of natural radioactivity and radiological hazards in construction materials used in Elazig, Turkey. Technical Report. Radiat. Meas. doi: 10.1016 / Jour. Radmeas. 2010.08.010.

Beretka, J. and Matthew, P. J. (1985): Natural radioactivity of Australian building materials, industrial wastes and by-products. Health Phys 1985 Jan; 48 (1):87-95. doi: 10.1097/00004032-198501000-00007.

Barker, F. (1979): Trondhjemite definition, environment and hypothesis of origin, El Sevier, Amsterdam, p. 1-12.

Berry, L. G., Brain, M. and Dietrich, R. V. (2000): Mineralogy. Concepts, descriptions and determinations. CBS Publishers and distributors, New Delhi (India). 561 p.

Briqueu, L., Bougault, H. and Joron, J. L. (1984): Quantification of Nb, Ta, Ti and V anomalies in magmas associated with subduction zones: Petrogenetic implications, Earth planet. Sci. Lett., V. 68, p. 297 - 308.

Cambon, A. R. (1994): Uranium deposits in granitic rocks. Notes on the national training course on uranium geology and exploration. Organized by IAEA and NMA, 8 - 20 Jan. 1994.

Cathelineau, M. and Holliger, P. (1987): Poly phase metallogensis of hydrothermal uranium veins from the southern Armorican Massif, France. Proc. Int. Mtg Nancy: P. 212-217.

Chapman, C. A. and Hall, A. (1997): The nature and origin of granite. Boundary Row, London. $387 \mathrm{p}$.

Chappell, B. W. and White, A. J. R. (1974): Two contrasting granite types, Pacific Geol., V. 8, p. 173-174.

Condie, K. C. (1973): Archean magmatism and crustal thickening. Geol. Soc. Amer. Bull., V. 84, p. 2981-2991.

Cuney, M. (1984): Les methods des prospection de l'uranium, Nuclear Energy Agency of the OECD, Paris. p. 277-292.

Deer, W. A., Howie, R. A. and Zussman, J. (1966): An introduction to rock forming minerals. Longmans, London. 517 p.

El Aassy, I. E., Nada, A. A., El-Galy, M. M. and El-Feky, M. G. (2012): Behavior and environmental impacts of radionuclides during the hydrometallurgy of calcareous and argillaceous rocks, southwestern Sinai, Egypt. , Applied radiation and isotopes, 70(6):1024-33.

El Galy, M. M., El Mezayn, A. M., Said, A. F., El Moafy, A. A. and Mohamed, M. S. (2008): Distribution and environmental impacts of some radionuclides in sedimentary rocks at Wadi Naseib area, southwest Sinai, Egypt. $\square \quad \square$ Journal of Environmental Radioactivity 99(7), 1075-82. 
Ford, K. L. (1982): Uraneferous pegmatites of the Sharbot Lake area, Ontario; in uranium in granites, ed. Y.T. Maurice; Geol Survey of Canada, 81-23, 125-138.

Harpy, N. M., El Dabour, S. E., Sallam, A. M., Nada, A. A., El Aassy, A. E. and ElFeky, M. G. (2019b): Environmental forensics, doi:10.1080/15275922.2019.1695020.

Hassan, M. A. and Hashad, A. H. (1990): Precambrian of Egypt. In: Said, R. (ed). The geology of Egypt, Balkema, Rotterdam, p. 201 - 245.

Heikal, M. T. S., Moharem, A. F. and El-Nashar, E. R. (2001): Petrogenesis and radioactive inspection of Li-mica pegmatites at Wadi Zareib, central Eastern Desert, Egypt. The second international conference on the Geology of Africa, Assiut, Oct. 2001, vol. II, p. 227-305.

Irvine, T.N., and Baragar, W.R.A. (1971): A guide to the chemical classification of the common volcanic rocks. Canada. J. Earth Sci.,V.8, p.523-548.

Lakehal, C. H., Ramdhan, M. and Boucenna, A. (2010): Natural radionuclide concentrations in two phosphate ores of east Algeria. Journal of Environmental Radioactivity, 101(5):377-9.

Lasheen, E. R. (2019): Petrology, Geochemistry of the Pan-African rocks at Soul Hamed area, South Eastern desert, Egypt and their suitability for some industrial applications, $\mathrm{PhD}$ thesis, AL- Azhar university, 329P.

Linnen, R. L. and Keppler, H. (1997): Colunbite solubility in granitic melts: consequences for the enrichment and fractionation of $\mathrm{Nb}$ and $\mathrm{Ta}$ in the earth's crust. Contrib. Mineral. Petrol. V. 128, p. 213-227.

Maurice, Y. T. (1982): Uranium in granites. Ottawa, Ontario, Canada. 172 p.

Mahdy M. A., Assaf, S. H., Abdel Kader, Z. M. and Omar, S. A (1994): Geological and geochemical features of radioactive occurrences in Gebel Ras Baroud, central Eastern Desert, Egypt. Egy. Min. V. 6, p. 167-184.

Mason B. (1966): Pyrope, augite, and hornblende from Kakanut, New Zeland: New Zeland Jour. Geology and Geophysics, V. 9, No. 4, p. 474-480.

Moharem, A. F. (2006a): Geochemistry and radio-elements distribution in the Suwayqat El-Arsha - Abu Ashayir granitic plutons and their associated pegmatites, central Eastern Desert, Egypt. Proceedings of the $9^{\text {th }}$ Arab Conf. for mineral resources (Kingdom of Saudi Arabia - Jeddah), Oct. 2006, vol. III, p. 246-264.

Moharem, A.F., (2006b) Uranium distribution in the mineral constituents of Um Samra - Um Bakra granitic plutons, central Eastern Desert, Egypt. Proceedings of the $7^{\text {th }}$ Arab Conf. on the peaceful uses of Atomic Energy, Dec. 2004, vol. II, 111- 125.

Nada, A. (2003): Evaluation of natural radionuclides at Um-Greifat area, Eastern Desert of Egypt. Appl. Radiat. Isot. 58, 275-280. 
NEA-OECD (1979): Exposure to Radiation from Natural Radioactivity in Building Materials. Report by NEA Group of Experts of the Nuclear Energy Agency, OECD, Paris, France.

Örgün Y., Altınsoy N., Ahin S. Y., Güngör Y., Gültekin A. H., Karahan G., Karacık Z. (2007): Natural and anthropogenic radionuclides in rocks and beach sands from Ezine region (Canakkale), Western Anatolia, Turkey. App. L Radiat. Isot 65:739-747.

Omar, S. A. (1995): Geological and geochemical features of the radioactive occurrences, south Gabal Um Anab granitic masses, Eastern Desert, Egypt. Ph. D Thesis, Faculty of Science, Cairo University, Cairo, Egypt, 164 p.

Raslan, M. F., El-Shall, H. E., Omar, S. A. and Daher, A. M. (2010): Mineralogy of polymetallic mineralized pegmatite of Ras Baroud granite, Central Eastern Desert, Egypt. Journal of Mineralogical and Petrological Sciences, Volume 105, P. 123134.

Read, H. H. (1984): Rutley's elements of mineralogy. Published by S. K. Jain for CBS Publishers and Distributors, Delhi, India, 560p.

Rismaite, J. (1977): Mineral assemblages at the, Rabbit Lack uranium deposit, Sastchwan, Geol. Surv. Can., V. IB, p. 235-246.

Rogers, J. J. W., Ghuma, M. A., Nagy, R. M., Greenberg, J. K. and Fullagar, P. D. (1978): Plutonism in Pan-African belts and the geologic evolution of north eastern Africa. Earth Planet Sci. Lett., V. 39, P. 109 - 117.

Saito, K. and Jacob, P. (1995): Gamma Ray Fields in the Air due to Sources in the Ground. Radiation Protection Dosimetry, 58, 29-45.

Scheepers, R. (2000): Granites of the Saldania mobile belt, South Africa: radioelements and $\mathrm{P}$ as discriminators applied to metallogeny. Journal of Geoch. Exp., V. 68, p 69-86.

Shalaby, M. H. (1995): New occurrence of uranium mineralizations G VII, Gabal Qattar uranium prospect, north Eastern Desert, Egypt. Bull. Fac. Sci. Alex. Univ., V.35, No.2, p.447-460.

Shalaby, M. H. and Moharem, A. F. (2001): Geochemistry and radioelement distribution in the fresh and altered Hammamat sedimentary rocks along Wadi Belih, southern Gabal Um Tawat, North Eastern Desert, Egypt. Sedimentology Soc. of Egypt. V. 9, p. 145-155. 
Shata, A. S., El-Husseiny, M. O. and Abu-Zeid, E. K. (2008): Petrography, mineralogy and geochemistry of the younger granites, Wadi Yahmid area, south east Sinai, Egypt (In Press)

Streckeisen, A. (1976): To each plutonic rock its proper name. Earth Sci. Rev., V. 12, p. 1-33.

Streckeisen, A. and Le Maitre, R. W. (1979): “A chemical approximation to the model QAPF classification of the igneous rocks" Neus Jahrb. Mineral. Abh., V. 136, p. 169-206.

Sylvester, A. G. (1980): Analysis of geodetic information, app. C. of Final geoseismic investigation, proposed LNG Terminal, little Cojo Ba, California: Dames and Moore, Inc., unpub. Report to Western LNG Terminal Association Inc., p. C-1 to C-20.

Taha, S. H., Sallam, O. R., Abbas, A. A. and Abed, N. S. (2020): Radioactivity and environmental impacts of ferruginous sandstone and its associating soil. International J. of Environmental Analytical Chemistry, 1-10.

Tufail, M., Siddiqui, M. S., Ali, S. and Mirza, N. M. (1992): Investigation of gammaray activity and radiological hazards of the bricks fabricated around Lahore (Pakistan). Pakistan journal of scientific and industrial research, 34(6), 216-220.

UNSCEAR (2000): Sources and effects of ionizing radiation. Report to the General Assembly of the United Nations with Scientific Annexes, United Nations sales publications.

White, A.J.R. and Chappell, B.W. (1983): Granitoid types and their distribution in the Lachlan Fold Belt, Southeastern Australia, Geol. Soc. Mem., V. 159, p. 21-34.

Winkler, H. G. F., Boese, M. and Macropoulos, T. (1975): Low temperature granitic melts. N. Jb. Mineral., V. 6, p. 245-268. 
الاشعاعية البيئية للبجماتيت المصاحب للجرانيت الحديث بمنطقة رأس بارود، وسط الصحراء الثرقية، مصر

\author{
اعداد

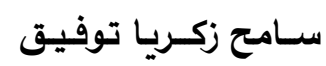 \\ هيئة المواد النووية، القاهرة، مصر توفي
}

الملخص باللغة العربية

تقع منطقة جبل رأس بارود فى وسط الصحراء الثرقية المصرية. تتواجد عروق البجماتيت فى منطقة الدراسة بجنوب جبل رأس بارود قاطعة صخور الجرانيت الحديث التي تتواجد بالمنطقة على هيئة بلوتونات دائرية وشبه دائرية منعزلة. والصخور البجماتيتية نوعان نطاقي و غير نطاقي. والنوع الأول يتواجد فى المرتفعات فقط بينما النوع الثانى يتواجد على الحدود الجنوبية لجبل راس بارود. البجماتيت النطاقي عبارة عن نطاق داخلى يتكون من الكوارتز ثم الفلسبار ثم الميكا (المسكوفيت مع وجود البيوتيت احياناً). ونطاق التغير يتواجد على حدود الكوارتز مع الميكا. يوجد بين النطاق اللبى والنطاق المتوسط نطاق تحلل يحتوي

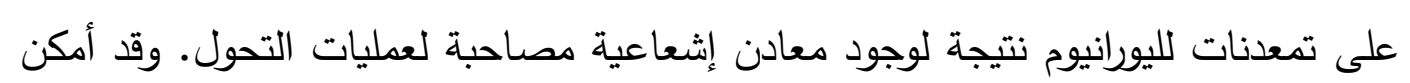
أكتشاف بعض من المعادن الثانوية لليورانيوم بهذا النطاق مثل البيتافيت والكازوليت. ويرجح نشأة تلك المعادن المشعة الى عمل المحاليل المائية الحارة التى لاتزيد درجة حرارتها عن 500 بحساب المعاملات البيئية الاشعاعية تبين لنا خطورة استخدام معادن نطاقات البجماتيت الأربعة لتجاوزها الحد الاثعاعي الآمن. كما بينت حسابات محتوى اليورانيوم الأولي واتجاهات هجرة اليورانيوم ومعدل هذه الهجرة أن اليورانيوم يهاجر من نطاق التحلل عالي الاشعاع الى نطاقات البجماتيت الأخرى (الخارجي والأوسط والداخلي) مما يعزز فكرة خطورة استخدام معادن هذه النطاقات للبناء او الزينة الا خارج المباني لتجنب تجمع غاز الرادون المشع. كما يجب تخزين هذه المواد ومنتجاتها في مستودعات جيدة التهوية ويتم التعامل معها بحذر من المتعاملين بها. 